\title{
Kaon and pion parton distributions
}

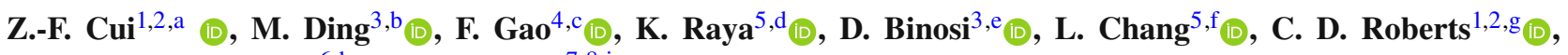 \\ J. Rodríguez-Quintero ${ }^{6, h}$, S. M. Schmidt ${ }^{7,8, i}$ \\ ${ }^{1}$ School of Physics, Nanjing University, Nanjing 210093, Jiangsu, China \\ ${ }^{2}$ Institute for Nonperturbative Physics, Nanjing University, Nanjing 210093, Jiangsu, China \\ ${ }^{3}$ European Centre for Theoretical Studies in Nuclear Physics and Related Areas (ECT*) and Fondazione Bruno Kessler, Villa Tambosi, \\ Strada delle Tabarelle 286, 38123 Villazzano, TN, Italy \\ ${ }^{4}$ Institut für Theoretische Physik, Universität Heidelberg, Philosophenweg 16, 69120 Heidelberg, Germany \\ ${ }^{5}$ School of Physics, Nankai University, Tianjin 300071, China \\ ${ }^{6}$ Department of Integrated Sciences and Center for Advanced Studies in Physics, Mathematics and Computation, University of Huelva, \\ 21071 Huelva, Spain \\ ${ }^{7}$ Helmholtz-Zentrum Dresden-Rossendorf, 01314 Dresden, Germany \\ ${ }^{8}$ RWTH Aachen University, III. Physikalisches Institut B, 52074 Aachen, Germany
}

Received: 1 July 2020 / Accepted: 20 October 2020 / Published online: 18 November 2020

(C) The Author(s) 2020

\begin{abstract}
Beginning with results for the leading-twist twoparticle distribution amplitudes of $\pi$ - and $K$-mesons, each of which exhibits dilation driven by the mechanism responsible for the emergence of hadronic mass, we develop parameterfree predictions for the pointwise behaviour of all $\pi$ and $K$ distribution functions (DFs), including glue and sea. The large- $x$ behaviour of each DF meets expectations based on quantum chromodynamics; the valence-quark distributions match extractions from available data, including the pion case when threshold resummation effects are included; and at $\zeta_{5}=5.2 \mathrm{GeV}$, the scale of existing measurements, the light-front momentum of these hadrons is shared as follows: $\left\langle x_{\text {valence }}\right\rangle^{\pi}=0.41(4),\left\langle x_{\text {glue }}\right\rangle^{\pi}=0.45(2),\left\langle x_{\text {sea }}\right\rangle^{\pi}=$ $0.14(2)$; and $\left\langle x_{\text {valence }}\right\rangle^{K}=0.42(3),\left\langle x_{\text {glue }}\right\rangle^{K}=0.44(2)$, $\left\langle x_{\text {sea }}\right\rangle^{K}=0.14(2)$. The kaon's glue and sea distributions are similar to those in the pion, although the inclusion of mass-dependent splitting functions introduces some differences on the valence-quark domain. This study should stimulate improved analyses of existing data and motivate new experiments sensitive to all $\pi$ and $K$ DFs. With little known empirically about the structure of the Standard Model's
\end{abstract}

\footnotetext{
a e-mail: phycui@nju.edu.cn

be-mail: mding@ectstar.eu

c e-mail: f.gao@thphys.uni-heidelberg.de

de-mail: khepani@nankai.edu.cn

e e-mail: binosi@ectstar.eu

f e-mail: leichang@nankai.edu.cn

ge-mail: cdroberts@nju.edu.cn (corresponding author)

he-mail: jose.rodriguez@dfaie.uhu.es

i e-mail: s.schmidt@hzdr.de
}

(pseudo-) Nambu-Goldstone modes and analyses of existing, limited data being controversial, it is likely that new generation experiments at upgraded and anticipated facilities will provide the information needed to resolve the puzzles and complete the picture of these complex bound states.

\section{Introduction}

The past decade saw discovery of the Higgs boson [1,2], thereby completing the Standard Model (SM). Nevertheless, two concrete paths remain open: searching for an understanding of known phenomena whose explanation is not provided by the SM, e.g. dark energy, dark matter, the size of the matter-antimatter asymmetry in the known Universe, etc. [3]; and elucidating the complete content and range of consequences of the SM.

The first track needs no explanation. This is a typical mode for high-energy physics. On the other hand, the second might seem odd. Consider, therefore, that although the SM is remarkably successful, it is only understood at the level of perturbation theory; and even in this context, e.g. the computation of five-point two-loop diagrams is an open challenge [4]. Moreover, there are SM phenomena whose explanation cannot be found in perturbation theory. Primary amongst these is the source of nuclear-size mass. The Higgs boson produces the Lagrangian (current) masses of all fermions; but so far as the building blocks of known nuclei are concerned (protons and neutrons, i.e. nucleons), the current masses of the valence $u$ - and $d$-quarks sum to just $1 \%$ of the nucleon mass. The remaining $99 \%$ is suspected to emerge as a conse- 
quence of dynamics within the SM's strong interaction sector; namely, quantum chromodynamics (QCD).

Emergent hadronic mass (EHM) is the origin of the $m_{N} \approx 1 \mathrm{GeV}$ scale that characterises and supports the bulk of visible matter; and any theoretical account should link EHM with an array of empirically verifiable consequences so that the explanation's credibility can be established. This requires a nonperturbative analysis of QCD in its strongcoupling domain. Critically, given the necessary connection between EHM and dynamical chiral symmetry breaking (DCSB), one should preferably employ nonperturbative methods in quantum field theory which preserve the WardGreen-Takahashi identities that connect symmetry-related Schwinger functions. With sufficient computer resources and careful analyses of the various necessary limits, numerical simulations of lattice-regularised QCD (1QCD) can meet this challenge [5-7]. An alternative approach is provided by continuum Schwinger function methods (CSMs); in particular, the Dyson-Schwinger equations (DSEs) [8-11], which provide a systematic, symmetry-preserving approach to solving the continuum bound-state problem for hadrons.

Significantly, where fair comparisons can be drawn, predictions from DSE analyses are practically identical to those obtained using 1QCD; and whilst the lattice formulation maintains a tighter connection with the QCD Lagrangian, the range of observables currently accessible to DSE methods is greater. The approaches are complementary and the synergy between them can profitably be exploited to understand EHM.

The need for a symmetry preserving framework is acute when studying pseudoscalar mesons whose valence constituents are drawn exclusively from the set of lighter $u-, d$-, $s$-quarks and their antiquark partners, e.g. $\pi$ and $K$ mesons. ${ }^{1}$ These states are massless in the absence of a Higgs mechanism: they are the SM's Nambu-Goldstone (NG) modes. As such, $\pi$ and $K$ mesons express a peculiar dichotomy. Namely, they are hadron bound states defined, like all others, by their valence quark and/or antiquark content; yet, the mechanism(s) which give all other hadrons their roughly $1 \mathrm{GeV}$ mass-scale are obscured in such systems. Hence, in exploring the origins of EHM, understanding the character of NG modes is of great importance [13]. These states are not pointlike; their internal structure is more complex than is usually imagined; and it can be argued that the properties of these nearly-massless strong-interaction composites provide the clearest windows onto EHM [14].

With the strong link to EHM in mind, in Sect. 2 we consider the simplest $\pi$ and $K$ distribution amplitudes (DAs), explain how they may be calculated, and draw a connection

\footnotetext{
${ }^{1}$ Owing to the non-Abelian anomaly, $\eta$ - and $\eta^{\prime}$-mesons are special cases with modified Ward-Green-Takahashi identities [12], which further complicate analyses.
}

between these DAs and the valence-quark distribution functions (DFs) of the $\pi$ and $K$. That connection is made through the light-front wave functions for these systems, which can be obtained via light-front projection of the appropriate BetheSalpeter wave functions. The key conjecture in Sect. 2 is that there exists a resolving scale, $\zeta_{H}$, at which the dressed quasiparticles emerging from the valence-quark and -antiquark degrees of freedom express all properties of the $\pi$ and $K$ mesons; in particular, they carry all the hadron's light-front momentum. (This feature is also typical of well-constructed models, e.g. Refs. [15-19].)

Section 3 reviews a perspective on QCD's running coupling, highlighting that the emergence of a gluon mass-scale [20-33] ensures QCD interactions can be described by a process-independent $(\mathrm{PI})$ effective charge which saturates to a nonzero, finite value at infrared momenta [34-38]. Such behaviour may be associated with the opening of a conformal window at long wavelengths. The associated gauge sector screening mass then serves as a natural value for $\zeta_{H}$ because it marks the border between soft and hard physics. In having thus identified $\zeta_{H}$ at the outset, all DA and DF results obtained herein are unified predictions $[39,40]$.

Our approach fixes $\zeta_{H}<m_{N}$; hence, comparisons with typical extractions of DFs require evolution to $\zeta>\zeta_{H}$. Section 4 therefore explains why the PI effective charge provides a suitable starting point for integrating the DGLAP equations [41-44], leading to an all-orders evolution scheme that enables predictions to be made for $\pi$ and $K$ DFs, i.e. valence, glue and sea. It also reiterates a longstanding prediction of the QCD-improved parton model [45-48], viz. in a $J=0$ hadron, $M$, the valence-quark DF has the following large- $x$ behaviour:

$q^{M}\left(x ; \zeta_{H}\right) \stackrel{x \cong 1}{=} c\left(\zeta_{H}\right)(1-x)^{\beta\left(\zeta_{H}\right)}, \beta\left(\zeta_{H}\right)=2$,

where $c\left(\zeta_{H}\right)$ is independent of $x$. Eq. (1) is controversial because most analyses of extant data on $q^{\pi}(x ; \zeta)$ imply $\beta\left(\zeta_{H}\right)<2$. The only analyses that agree with Eq.(1) are those at next-to-leading order (NLO) which include threshold resummation effects $[49,50]$. Much of the discussion herein bears upon the validity of Eq. (1).

Section 5 presents our results for $\pi$ DFs, providing extensive comparisons, e.g.: with older [51] and more recent phenomenological analyses [52]; and with recent 1QCD results for the moments [53,54] and pointwise behaviour [55] of $q^{\pi}(x)$.

Our prediction for the kaon's simplest DA is explained in Sect. 6. Building upon that, Sect. 7 describes predictions for all the kaon's DFs. They include results for the hostdependence of valence, glue and sea DFs, i.e. $K / \pi$ ratios for all common DFs. It should be emphasised that within the SM's strong interaction sector there can be no differences between $\pi$ and $K$ mesons without a Higgs mechanism; but EHM modulates the observable effects of this mechanism in a 
variety of ways, many of which have not yet been elucidated. Finally, along with providing a summary, Sect. 8 presents a perspective on the potential for further studies of $\pi$ and $K$ structure to deliver insights into EHM and the manner by which its effects are tempered by the Higgs boson.

\section{Light front wave functions and parton distributions}

If one seeks a description of a given hadron's measurable properties in terms of the probability expressions typical of quantum mechanics, then the hadron's light front wave function (LFWF), $\psi_{H}\left(x, \mathbf{k}_{\perp} ; P\right)$, where $P$ is the hadron's total momentum, takes a leading role. In approaching the problem of expressing $\psi$ from a partonic perspective with a connection to perturbative QCD, it is usual to employ a Fock-space decomposition of the LFWF. Each element in that expansion then represents the probability amplitude for finding $n$ partons in the hadron with momenta $\left\{\left(x_{i}, k_{\perp i}\right) \mid i=1, \ldots, n\right\}$, always requiring conservation of total momentum.

The strength of this approach is exemplified by observing that the DAs which feature in formulae describing hard exclusive processes and the DFs that characterise hard inclusive reactions can both be expressed directly in terms of the LFWF [56], viz., respectively:

$$
\begin{aligned}
& \varphi_{H}(x ; \zeta) \propto \int^{\zeta} d^{2} k_{\perp} \psi_{H}\left(x, \mathbf{k}_{\perp} ; P\right), \\
& q^{H}(x ; \zeta) \propto \int^{\zeta} d^{2} k_{\perp}\left|\psi_{H}\left(x, \mathbf{k}_{\perp} ; P\right)\right|^{2},
\end{aligned}
$$

where $\zeta$ is the scale at which the hadron is being resolved. As indicated by this $\zeta$-dependence, considered as a problem in quantum field theory, the perceived character of any hadron depends upon the energy scale at which it is observed. That is not to say empirical cross-sections are affected; rather, this energy scale determines the optimal choice for the degrees of freedom needed to solve the problem and express an insightful interpretation.

In order to capitalise on Eq. (2), one must be able to compute a hadron's LFWF. This might be achieved by constructing a sound approximation to QCD's light-front Hamiltonian [57]. However, that path is complicated by, inter alia, the need to solve complex constraint equations along the way [58]. A different approach, applied elsewhere [59] to a local $\mathrm{U}\left(N_{c}\right)$ gauge theory in two dimensions, with $N_{c}$ very large, is to: use the covariant DSEs; solve for Bethe-Salpeter wave functions; and subsequently project these quantities onto the light front, thereby obtaining the desired LFWFs. This scheme was shown to be practical for QCD in Ref. [60]. We follow it herein.

In employing DSEs to solve the continuum bound state problem, one must specify a kernel. In most cases, a rainbow- ladder (RL) truncation is used, viz. leading order in the systematic, symmetry-preserving scheme formalised in Refs. $[61,62]$. For instance, RL studies have recently delivered predictions for the valence, glue and sea distributions within the pion $[39,40]$ and unified them with, e.g. electromagnetic pion elastic and transition form factors [63-66].

Experience and practice have shown that owing to cancellations between correction terms, RL truncation delivers sound predictions for diverse properties of systems constituted from (nearly) degenerate valence-parton degrees-offreedom in which orbital angular momentum does not play a significant role, e.g. [67-73]: $\pi$ and $\rho$ mesons and the nucleon and $\Delta$-baryon. However, in mesons with an imbalance between the current-masses of the valence-quark and antiquark, straightforward implementation of RL truncation becomes increasingly unreliable as the difference between current masses increases. For instance, concerning the kaon, it yields a leading-twist DA, $\varphi_{K}(x)$, that is both too asymmetric around $x=1 / 2$ and too dilated [74,75]. Many such defects are remedied by the dynamical-chiral-symmetrybreaking-improved (DB) truncation [76], whose development enabled a bridge to be built between studies of QCD's gauge and matter sectors [28].

QCD is a renormalisable quantum field theory; hence, a renormalisation scheme and scale must be chosen when formulating and solving the continuum bound state problem. We use a mass-independent momentum subtraction procedure with scale $\zeta=\zeta_{H}$, i.e. the infrared "hadronic" scale at which the dressed quasiparticles obtained as solutions to the quark gap equation express all properties of the bound state under consideration, e.g. they carry all of the hadron's momentum at $\zeta_{H}$. As explained elsewhere [39,40,64,65,77,78], this approach ensures that parton splitting can properly be expressed through $\zeta$-evolution of hadron wave functions [7981] thereby overcoming a known deficiency of truncated bound-state kernels. (This is elucidated in Sect. 5.1.)

Following the procedure just described, reviewed in Refs. [8-10], one can obtain the Bethe-Salpeter wave functions for the $\pi$ and $K$ described as bound states of a dressed $u$-quark and $h$-antiquark $(M=\pi, h=d$ and $M=K, h=s)$ :

$\chi_{M}^{P}\left(k_{\eta \bar{\eta}} ; \zeta_{H}\right)=S_{u}\left(k_{\eta} ; \zeta_{H}\right) \Gamma_{M}^{P}\left(k_{\eta \bar{\eta}} ; \zeta_{H}\right) S_{h}\left(k_{\bar{\eta}} ; \zeta_{H}\right)$,

where $P$ is the meson's total momentum; $S_{f}\left(k ; \zeta_{H}\right)$ is the dressed propagator for a quark with flavour $f$ and renormalisation point invariant current-mass $\hat{m}_{f}$,

$S_{f}\left(k ; \zeta_{H}\right)=Z_{f}\left(k^{2} ; \zeta_{H}^{2}\right) /\left[i \gamma \cdot k+M_{f}\left(k^{2}\right)\right] ;$

and the Bethe-Salpeter amplitude $\left(\ell=k_{\eta \bar{\eta}}\right)$

$$
\begin{gathered}
\Gamma_{M}^{P}\left(\ell ; \zeta_{H}\right)=\gamma_{5}\left[i E_{M}\left(\ell ; P ; \zeta_{H}\right)+\gamma \cdot P F_{M}\left(\ell ; P ; \zeta_{H}\right)\right. \\
\left.\quad+\gamma \cdot \ell G_{M}\left(\ell ; P ; \zeta_{H}\right)+\sigma_{\mu \nu} \ell_{\mu} P_{\nu} H_{M}\left(\ell ; P ; \zeta_{H}\right)\right]
\end{gathered}
$$


$E_{M}\left(\ell ; P ; \zeta_{H}\right)$ is a scalar function of $\ell^{2}, \ell \cdot P$; likewise, the other functions. Here, $k_{\eta \bar{\eta}}=\left[k_{\eta}+k_{\bar{\eta}}\right] / 2, k_{\eta}=k+\eta P$, $k_{\bar{\eta}}=k-(1-\eta) P, \eta \in[0,1]$; and owing to Poincaré covariance, no observable depends on $\eta$, i.e. the definition of the relative momentum. (The analysis herein is predicated on the following current-quark mass values: $\hat{m}_{u}=\hat{m}_{d}=$ 4.4 MeV, $\hat{m}_{s}=90 \mathrm{MeV}$.)

It is worth highlighting that the matrix valued function $\chi_{M}^{P}\left(k_{\eta \bar{\eta}} ; \zeta_{H}\right)$, described above, yields a LFWF for the meson $M$ whose Fock space expansion in terms of non-interacting parton eigenstates of the free light-front Hamiltonian contains a countable infinity of terms. In practice, the weighting of each one of the terms in the LFWF is determined by the truncation employed in the DSE calculation; and as the truncation is improved, these strengths more veraciously express QCD.

The leading-twist two-particle DA for the $u$-quark in the meson $M$ can now be obtained by light-front projection of $\chi_{M}$ :

$$
\begin{aligned}
& f_{M} \varphi_{M}^{u}\left(x ; \zeta_{H}\right)=\frac{1}{16 \pi^{3}} \int d^{2} k_{\perp} \psi_{M_{u}}^{\uparrow \downarrow}\left(x, k_{\perp}^{2} ; \zeta_{H}\right) \\
& =N_{c} \operatorname{tr} Z_{2}\left(\zeta_{H}, \Lambda\right) \int_{d k}^{\Lambda} \delta_{n}^{x}\left(k_{\eta}\right) \gamma_{5} \gamma \cdot n \chi_{M}\left(k_{\eta \bar{\eta}} ; P ; \zeta_{H}\right),
\end{aligned}
$$

where $N_{c}=3$; the trace is over spinor indices; $\int_{d k}^{\Lambda}$ is a symmetry-preserving regularisation of the four-dimensional integral, with $\Lambda$ the regularisation scale; $Z_{2}\left(\zeta_{H}, \Lambda\right)$ is the quark wave function renormalisation constant; $\delta_{n}^{x}\left(k_{\eta}\right)=\delta(n$. $\left.k_{\eta}-x n \cdot P\right), n$ is a light-like four-vector, $n^{2}=0$, with $n \cdot P=-m_{M}$ in the meson rest frame; and $f_{M}$ is the meson's leptonic decay constant, so

$\int_{0}^{1} d x \varphi_{M}^{u}\left(x ; \zeta_{H}\right)=1$

The companion DA for the $h$-antiquark is

$\varphi_{M}^{\bar{h}}\left(x ; \zeta_{H}\right)=\varphi_{M}^{u}\left(1-x ; \zeta_{H}\right)$.

In terms of the two-particle LFWF defined via Eq. (6a), the meson's valence $u$-quark DF is

$u^{M}\left(x ; \zeta_{H}\right)=\int d^{2} k_{\perp}\left|\psi_{M_{u}}^{\uparrow \downarrow}\left(x, k_{\perp}^{2} ; \zeta_{H}\right)\right|^{2}$.

This can be demonstrated in many ways, one of which proceeds from analysis of pseudoscalar meson generalised parton distributions [82]. As explained above, since the meson $M$ is constituted solely from dressed $u$ and $\bar{h}$ degrees-offreedom at $\zeta_{H}$, then

$\bar{f}^{M}\left(x ; \zeta_{H}\right)=u^{M}\left(1-x ; \zeta_{H}\right)$.

At this point we recall the analysis of $\pi$ and $K$ distributions in Ref. [83], which demonstrates that a factorised representation of $\psi_{M_{u}}^{\uparrow \downarrow}\left(x, k_{\perp}^{2} ; \zeta_{H}\right)$ is quantitatively reliable for integrated quantities. Namely, it is a good approximation to write

$\psi_{M_{u}}^{\uparrow \downarrow}\left(x, k_{\perp}^{2} ; \zeta_{H}\right)=\varphi_{M}^{u}\left(x ; \zeta_{H}\right) \psi_{M_{u}}^{\uparrow \downarrow}\left(k_{\perp}^{2} ; \zeta_{H}\right)$,

where $\psi_{M_{u} \uparrow \downarrow}^{\uparrow}\left(k_{\perp}^{2} ; \zeta_{H}\right)$ is some sensibly chosen function. Using Eq. (9), it follows with equal precision that ${ }^{2}$

$u^{M}\left(x ; \zeta_{H}\right) \propto\left|\varphi_{M}^{u}\left(x ; \zeta_{H}\right)\right|^{2}$,

where the constant of proportionality is fixed by baryon number conservation. Owing to parton splitting effects, Eq. (12) is not valid on $\zeta>\zeta_{H}$. Nevertheless, since the evolution equations for both DFs and DAs are known [41-44,79-81], the connection changes in a traceable manner.

\section{Process independent effective charge and the hadronic scale}

In studies of DFs, the hadronic scale, $\zeta_{H}$, is typically used as a parameter [84]. A practitioner develops a DF model that is supposed to be valid at an unspecified scale, which is subsequently identified as $\zeta_{H}$. Then a target DF is identified, one that has typically been extracted through a phenomenological analysis of selected experimental data at $\zeta_{E} \gg \zeta_{H}$. The practitioner finally chooses a value of $\zeta_{H}$ so that, after DGLAP evolution $\zeta_{H} \rightarrow \zeta_{E}$, the model DF reproduces some property or properties of the target distribution.

We adopt a different approach, which delivers predictive power $[39,40]$. It capitalises on the existence of a PI effective charge in QCD [38], $\hat{\alpha}\left(k^{2}\right)$, which, owing to the emergence of a nonzero gluon mass-scale [20], saturates in the infrared: $\hat{\alpha}(0) / \pi=0.97(4)$. An interpolation of the numerical result is obtained by writing

$\hat{\alpha}\left(k^{2}\right)=\frac{\gamma_{m} \pi}{\ln \left[\frac{\mathcal{K}^{2}\left(k^{2}\right)}{\Lambda_{\mathrm{QCD}}^{2}}\right]}, \mathcal{K}^{2}(y)=\frac{a_{0}^{2}+a_{1} y+y^{2}}{b_{0}+y}$,

$\gamma_{m}=4 / \beta_{0}, \beta_{0}=11-(2 / 3) n_{f}, n_{f}=4, \Lambda_{\mathrm{QCD}}=$ $0.234 \mathrm{GeV}$, with (in $\mathrm{GeV}^{2}$ )

\begin{tabular}{c|c|c}
$a_{0}$ & $a_{1}$ & $b_{0}$ \\
\hline $0.104(1)$ & 0.0975 & $0.121(1)$
\end{tabular}.

This interpolation is depicted in Fig. 1. Notably, $\hat{\alpha}\left(k^{2}\right)$ agrees to better than $0.1 \%$ with QCD's one-loop perturbative running coupling on $k^{2} \gtrsim\left(9 \Lambda_{\mathrm{QCD}}\right)^{2}$.

The perturbative running coupling in QCD exhibits a Landau pole at $k^{2}=\Lambda_{\mathrm{QCD}}^{2}$. This singularity is eliminated

\footnotetext{
${ }^{2}$ In the limit of exact $\mathcal{G}$-parity symmetry, which is a good approximation in the Standard Model, $u^{\pi^{+}}(x)=\bar{d}^{\pi^{+}}(x)$, etc.
} 


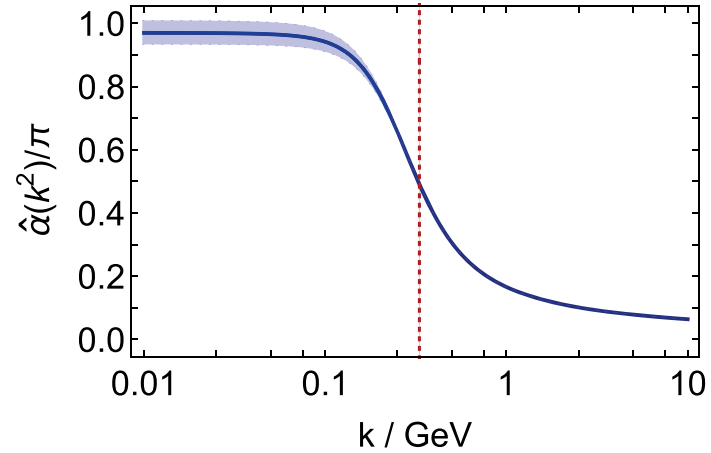

Fig. 1 Process-independent effective charge, $\hat{\alpha}\left(k^{2}\right) / \pi$, computed elsewhere [38] and interpolated using Eqs. (13), (14). (The band bracketing the solid blue curve expresses the uncertainty in $\hat{\alpha}\left(k^{2}=0\right) / \pi=$ $0.97(4)$. Details are provided in Ref. [38].) The vertical dashed red line marks the screening mass $k=m_{G}=0.331(2) \mathrm{GeV}$

from the PI effective charge by nonperturbative gauge sector dynamics. The effect of such dynamics is plain in Eq. (13), where $k^{2} / \Lambda_{\mathrm{QCD}}^{2}$ is replaced by $\mathcal{K}^{2}\left(k^{2}\right) / \Lambda_{\mathrm{QCD}}^{2}$ as the argument of the logarithm. The value

$m_{G}:=\mathcal{K}\left(k^{2}=\Lambda_{\mathrm{QCD}}^{2}\right)=0.331(2) \mathrm{GeV}$

thus defines a screening mass. As evident in Fig. 1, $m_{G}$ marks a boundary: the running coupling alters character at $k \simeq m_{G}$ so that modes with $k^{2} \lesssim m_{G}^{2}$ are screened from interactions and the theory enters a conformal domain. This being so, then the line $k=m_{G}$ draws a natural border between soft and hard physics; hence, we identify

$\zeta_{H}=m_{G}$

\section{Scale evolution of distribution functions}

\subsection{All-orders evolution hypothesis}

The hadronic scale, $\zeta_{H}$, is not directly accessible in analyses of experiments capable of providing information about DFs because certain kinematic conditions need to be met in order for the data to be interpreted in such terms [85]. These conditions typically require experiments with momentum transfers squared $Q^{2} \sim \zeta_{E}^{2}>m_{N}^{2}$. Hence, any result for a DF at $\zeta_{H}$ must be evolved to $\zeta_{E}$ for comparison with experiment.

The usual evolution framework is provided by the DGLAP equations. However, an evolution prescription must still be specified because the DGLAP equations involve QCD's running coupling. One may take a purely perturbative QCD (pQCD) perspective and implement evolution by using DGLAP kernels computed at a given order in perturbation theory. In this case, if the scale at which evolution begins is large enough, then leading-order (LO) evolution kernels may be sufficient, at least in practice. Failing that, then next-toleading order can be implemented, and so on, in principle.

An alternative supposes that [86-90]: (i) in connection with a given process, a nonperturbative running coupling (effective charge) exists; (ii) being derived from experiment, this charge is free of a Landau pole; and (iii) using this charge, the associated leading-order DGLAP equations are exact. Notwithstanding the feature that effective charges from different observables can in principle be related via an expansion of one coupling in terms of the other, the process-dependence of such couplings may be unsettling because: (a) knowledge of one process-dependent (PD) charge does not usually enable global predictions to be made for another process; and (b) the connection between two such charges at infrared momenta can only be determined after both are independently constructed.

Following Refs. [37-40], our approach is to refashion the PD-charge alternative and implement evolution by employing the PI effective charge described in Sect. 3, to integrate the one-loop DGLAP equations. Using this procedure, e.g. one has the following relationship between the Mellin moments of a meson's valence-quark DF:

$$
\left\langle x^{n} q^{M}\right\rangle_{\zeta}:=\int_{0}^{1} d x x^{n} q^{M}(x ; \zeta)
$$

$$
\begin{aligned}
x_{M}^{n}\left(\zeta_{H}, \zeta\right) & :=\frac{\left\langle x^{n} q^{M}\right\rangle_{\zeta}}{\left\langle x^{n} q^{M}\right\rangle_{\zeta_{H}}} \\
& =\exp \left[\frac{\gamma_{0}^{n}}{4 \pi} \int_{\ln \zeta^{2}}^{\ln \zeta_{H}^{2}} d t \hat{\alpha}(t)\right],
\end{aligned}
$$

where $t=\ln k^{2}$ and

$\gamma_{0}^{n}=-\frac{4}{3}\left[3+\frac{2}{(n+1)(n+2)}-4 \sum_{k=1}^{n+1} \frac{1}{k}\right]$.

Note that $\gamma_{0}^{0}=0$, so baryon-number does not change with $\zeta$, and $\gamma_{0}^{1}=32 / 9$.

Our reasons for adopting this scheme are manifold. For example, as illustrated in Fig. $1, \hat{\alpha}\left(k^{2}\right)$ is monotonically decreasing on $k^{2} \geq 0$ and capable of marking the boundary between soft and hard physics. Moreover, it is known to unify many observables, inter alia: hadron static properties [72,73,91,92]; parton distribution amplitudes of lightand heavy-mesons [74,75,93-95] and associated elastic and transition form factors $[66,77,78,96,97]$. In addition, $\hat{\alpha}\left(k^{2}\right)$ is pointwise (almost) identical to the PD effective charge, $\alpha_{g_{1}}$, defined via the Bjorken sum rule [98-100]. In possessing such an array of properties, $\hat{\alpha}\left(k^{2}\right)$ emerges as a strong candidate for that object which properly represents the inter- 
action strength in QCD at any given momentum scale [87]. Hence, it is a suitable input for our procedure. ${ }^{3}$

\subsection{Distribution functions on $x \simeq 1$}

The Introduction reiterated one of the earliest predictions of the QCD-improved parton model [45-48]:

$q^{M}\left(x ; \zeta_{H}\right) \stackrel{x \cong 1}{=} c\left(\zeta_{H}\right)(1-x)^{\beta\left(\zeta_{H}\right)}, \beta\left(\zeta_{H}\right)=2$,

where $c\left(\zeta_{H}\right)$ is a constant, i.e. independent of $x$, and the exponent increases logarithmically with $\zeta: \beta(\zeta)>\beta\left(\zeta_{H}\right)$ for $\zeta>\zeta_{H}$. In fact, as shown in Appendix 1, an analysis of the large- $n$ behaviour of Eqs. (17)-(19) yields $\left(\gamma_{E}=0.5772 \ldots\right.$ is Euler's constant)

$$
\begin{aligned}
& \beta(\zeta)=\beta\left(\zeta_{H}\right)+\frac{3}{2} \ln \chi_{M}^{1}\left(\zeta, \zeta_{H}\right), \\
& c(\zeta)=c\left(\zeta_{H}\right) \frac{\Gamma\left(1+\beta\left(\zeta_{H}\right)\right)}{\Gamma(1+\beta(\zeta))}\left[\chi_{M}^{1}\left(\zeta, \zeta_{H}\right)\right]^{\frac{3}{2}\left[\frac{3}{4}-\gamma_{E}\right]} .
\end{aligned}
$$

It is worth noting that although Eq. (21a), when expressed at one-loop order in pQCD, is a textbook result, e.g. Ref. [85, Eq. (4.137)], it is often overlooked. Equation (21) establish a connection between the large- $x$ exponent of a meson's valence-quark DF at scale $\zeta$ and the momentum fraction carried by valence-quarks at this scale, viz. the exponent increases logarithmically with the decrease in valencequark momentum fraction and the multiplicative coefficient decreases.

\section{Pion distributions}

\subsection{Scale invariance}

The DFs of valence-quarks, sea and glue in the pion were calculated using a symmetry-preserving implementation of RL truncation in Refs. $[39,40]$. In that study, the valencequark DF was obtained using [101]:

$$
\begin{aligned}
q^{\pi}(x ; \zeta)= & N_{c} \operatorname{tr} \int_{d k} \delta_{n}^{x}\left(k_{\eta}\right) \Gamma_{\pi}^{P}\left(k_{\bar{\eta} \eta} ; \zeta\right) S\left(k_{\bar{\eta}} ; \zeta\right) \\
& \times\left\{n \cdot \frac{\partial}{\partial k_{\eta}}\left[\Gamma_{\pi}^{-P}\left(k_{\eta \bar{\eta}} ; \zeta\right) S\left(k_{\eta} ; \zeta\right)\right]\right\} .
\end{aligned}
$$

This is a practicable approximation to the complete set of box diagrams necessary for a symmetry-preserving calculation of the valence-quark DFs. Using Eq. (22), it is straightforward to verify Eq. (10).

\footnotetext{
${ }^{3}$ Even if one chooses to doubt the implied global character of our evolution scheme, then given that $\hat{\alpha}\left(\zeta_{H}\right) /(2 \pi)=0.25,\left[\hat{\alpha}\left(\zeta_{H}\right) /(2 \pi)\right]^{2}=$ 0.06 , it can otherwise be viewed as expressing a self-stabilising oneloop approximation to the DGLAP equations.
}

All existing continuum quantum field theory studies of meson valence-quark DFs begin with a formula in the same class as Eq. (22) - see Ref. [48] and citations thereof. It is important, therefore, to highlight a particular feature of Eq. (22) that has hitherto been overlooked. Namely, computed using functions obtained in rainbow-ladder truncation or any kindred variant, the result is actually independent of $\zeta$.

The proof is straightforward. In a computational framework that preserves the multiplicative renormalisability of QCD:

$$
\begin{aligned}
S\left(k ; \zeta_{1}\right) Z_{2}\left(\zeta_{1}^{2}, \Lambda^{2}\right) & =S\left(k ; \zeta_{2}\right) Z_{2}\left(\zeta_{2}^{2}, \Lambda^{2}\right), \\
\Gamma^{P}\left(k_{\eta \bar{\eta}} ; \zeta_{1}\right) / Z_{2}\left(\zeta_{1}^{2}, \Lambda^{2}\right) & =\Gamma^{P}\left(k_{\eta \bar{\eta}} ; \zeta_{2}\right) / Z_{2}\left(\zeta_{2}^{2}, \Lambda^{2}\right) .
\end{aligned}
$$

Hence,

$S\left(k ; \zeta_{1}\right) \Gamma^{P}\left(k_{\eta \bar{\eta}} ; \zeta_{1}\right)=S\left(k ; \zeta_{2}\right) \Gamma^{P}\left(k_{\eta \bar{\eta}} ; \zeta_{2}\right)$

and, as defined by Eq. $(22), q^{\pi}\left(x ; \zeta_{1}\right)=q^{\pi}\left(x ; \zeta_{2}\right)$. It should be noted that the same is true of $\varphi_{M}(x ; \zeta)$ obtained from Eq. (6b).

This characteristic is good so far as baryon number conservation is concerned because it guarantees

$1=\int_{0}^{1} d x q^{\pi}\left(x ; \zeta_{1}\right)=\int_{0}^{1} d x q^{\pi}\left(x ; \zeta_{2}\right)$

However, it also imposes the same identity for all $n \geq 1$ moments (Eq. 17), i.e. it precludes DF evolution. Consequently, like Eq. (12), Eq. (22) can only be valid for $\zeta=\zeta_{H}$, whereat the meson is constituted solely from dressed $u$ and $\bar{h}$ degrees-of-freedom.

If one analyses the derivations of formulae in the class of truncations which contains Eq. (22), the origin of the scale invariance becomes clear. In fact, it was identified in Ref. [81]: diagrams with any number of longitudinally polarised gluons contribute at leading order in covariant gauges and they are neglected in deriving Eq. (22) and kindred expressions. Such contributions do not change power laws associated with scaling behaviour; but they do produce the anomalous dimensions necessary for scaling violations and parton splitting, i.e. the undressing of dressed quasiparticles as $\zeta$ is increased. Another way of understanding this is to recognise that any Wilson line is nonzero in calculations using covariant gauges; hence, the bare partial derivative in the light-front projection should be converted into the covariant derivative. It was to remedy precisely these weaknesses that Refs. $[64,65,77,78]$ incorporated Bethe-Salpeter wave function evolution. 


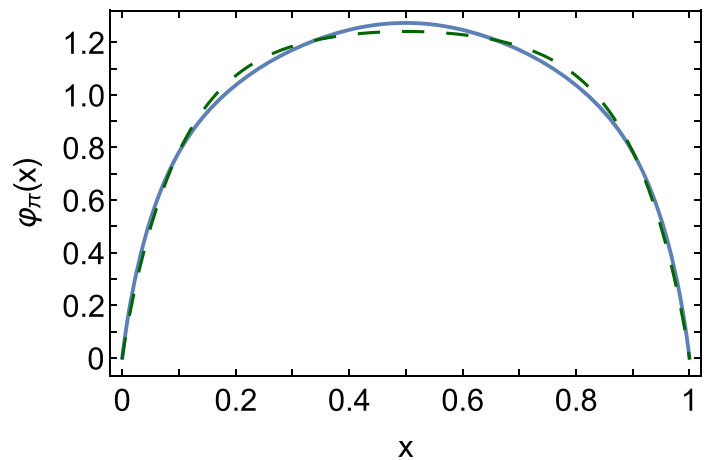

Fig. 2 Comparison of pion leading-twist two-particle DAs: $\varphi_{\pi}\left(x ; \zeta_{H}\right)$ computed using the DB kernel [60], solid blue curve; and Eq. (27), dashed green curve

\subsection{Connecting the pion's DF and DA}

In Refs. [39,40], the single mass-scale characterising the interaction used to define the RL truncation was chosen so as to deliver a realistic description of pseudoscalar mesons constituted from mass-degenerate valence-quark and -antiquark degrees-of-freedom over a wide range of current-quark masses [66]. It follows that implementing a DB kernel cannot bring material improvement because no realistic kernel can do better than reproduce experiment and the RL study was already tuned to that purpose.

The preceding claim and also the utility of Eq. (12) can be validated as follows. In Refs. [39,40] the valence-quark DF is

$$
\begin{aligned}
q_{\mathrm{O}}^{\pi}\left(x ; \zeta_{H}\right)= & 213.32 x^{2}(1-x)^{2} \\
& \times[1-2.9342 \sqrt{x(1-x)}+2.2911 x(1-x)],
\end{aligned}
$$

from which one obtains the unit-normalised square-root:

$$
\begin{aligned}
& \varphi_{\pi}^{\sqrt{ }}\left(x ; \zeta_{H}\right)=15.271 x(1-x) \\
& \quad \times[1-2.9342 \sqrt{x(1-x)}+2.2911 x(1-x)]^{1 / 2} .
\end{aligned}
$$

According to Eq. (12), $\varphi_{\pi}^{\sqrt{ }}$ should be a fair approximation to the pion's leading-twist two-particle DA; and this is verified by Fig. 2, which compares Eq. (27) with the result obtained from Eq. (6b) using the DB kernel [60]:

$$
\begin{aligned}
& \varphi_{\pi}^{\mathrm{DB}}\left(x ; \zeta_{H}\right)=20.227 x(1-x) \\
& \quad \times[1-2.5088 \sqrt{x(1-x)}+2.0250 x(1-x)] .
\end{aligned}
$$

It is necessary to remark here that Ref. [60] chose to reconstruct the pion's DA from its moments using an order$\alpha$ Gegenbauer expansion. This is a useful first step because the procedure converges rapidly; hence, enables the qualitative feature of broadening driven by EHM in the SM to be exposed. Such broadening, however, need not and should not disturb the DA's endpoint behaviour, which QCD predicts to be linear in the neighbourhoods $x \simeq 0,1$. Therefore, as a second step, we re-expressed the result from Ref. [60] as the function in Eq. (28). The first eleven moments agree at the level of 1.6(1.4)\%, i.e. well within any sensible estimate of uncertainty in the computation of high-order moments.

Hereafter, we consider Eqs. (12), (28) to deliver the best founded prediction for the pion's valence-quark DFs, viz.

$$
\begin{aligned}
& u^{\pi}\left(x ; \zeta_{H}\right)=375.32 x^{2}(1-x)^{2} \\
& \quad \times[1-2.5088 \sqrt{x(1-x)}+2.0250 x(1-x)]^{2},
\end{aligned}
$$

with $\bar{d}^{\pi}=u^{\pi}$. Notably, even though it is plain that

$u^{\pi}\left(x ; \zeta_{H}\right) \stackrel{x \cong 1}{=} 375.32(1-x)^{2}=: u_{1}^{\pi}\left(x ; \zeta_{H}\right)$,

$u^{\pi}\left(x ; \zeta_{H}\right) / u_{1}^{\pi}\left(x ; \zeta_{H}\right)>0.5$ is only realised on $x>0.98$ as a consequence of EHM-induced broadening.

\subsection{Evolved pion DFs}

\subsubsection{GRS scale}

Reference [51] (GRS) postulates that hadron DFs at a scale $\zeta$ can be computed by beginning with nonzero valence-like distributions for all partons at a scale $\zeta_{0}$ and then using pQCD DGLAP equations (at LO or NLO) to evolve these input distributions to the larger scale, $\zeta$, in the process of fitting a selected body of structure function data. There are similarities between this hypothesis and our approach, but also differences, e.g.: whilst Ref. [51] uses $\zeta_{0}$ as a parameter, our value of $\zeta_{H}$ is fixed by $\hat{\alpha}\left(k^{2}=\Lambda_{\mathrm{QCD}}^{2}\right)$; and Ref. [51] has nonzero distributions for all partons at the starting scale, $\zeta_{0}$, whereas we argue that glue and sea distributions are zero at $\zeta_{H}$. Notably, GRS use $\zeta_{0}^{\mathrm{LO}}=0.51 \mathrm{GeV}$ or $\zeta_{0}^{\mathrm{NLO}}=0.63 \mathrm{GeV}$ and we have $\zeta_{H}=0.33 \mathrm{GeV}<\zeta_{0}^{\mathrm{LO}}<\zeta_{0}^{\mathrm{NLO}}$. Given this feature, we judge it worthwhile to begin with Eq. (29), employ the evolution procedure explained in Sect.4.1, and compare our predictions for the pion's valence-quark, glue and sea distributions at a GRS scale with the input distributions assumed in Ref. [51]. Only the LO GRS distributions are reported. For all practical purposes, the comparison with NLO GRS is equivalent.

Our $\zeta_{H} \rightarrow \zeta_{0}^{\mathrm{LO}}$ evolved valence-quark DF is compared with the GRS Ansatz in Fig. 3a. Evidently, the GRS distribution is much harder than our prediction. Notwithstanding that, the valence-quark momentum fraction is comparable with ours, albeit 29(12)\% smaller: we predict

$$
\left\langle 2 x u^{\pi}\left(x ; \zeta_{0}^{\mathrm{LO}}\right)\right\rangle=0.73(7),
$$



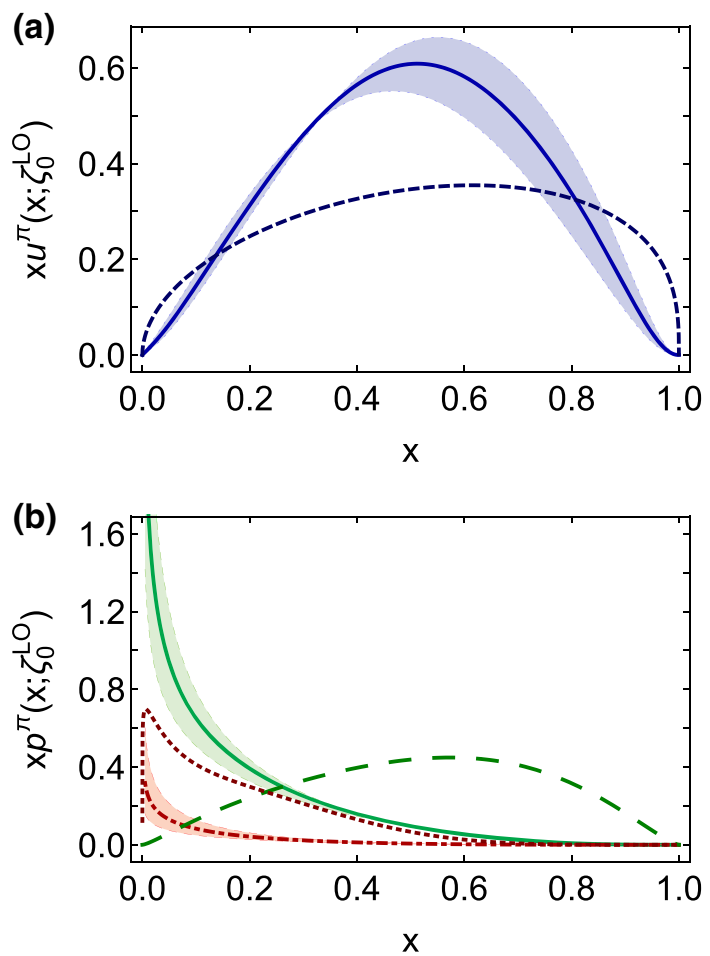

Fig. 3 a Solid blue curve: valence-quark distribution in Eq. (29) evolved to the GRS-input scale, $\zeta_{0}^{L O}=0.51 \mathrm{GeV}$, using the procedure explained in Sect. 4.1. Dashed blue curve: GRS input distribution at this scale [51]. b Solid green curve, $p=g$-our prediction for the pion's glue distribution; and dot-dashed red curve, $p=S$-predicted seaquark distribution. The long-dashed green curve and dotted red curve are the GRS input distributions: $p=g$ and $p=S$, respectively. In our normalisation convention, $\left\langle x\left[2 u^{\pi}\left(x ; \zeta_{0}^{L O}\right)+g^{\pi}\left(x ; \zeta_{0}^{L O}\right)+\right.\right.$ $\left.\left.S^{\pi}\left(x ; \zeta_{0}^{L O}\right)\right]\right\rangle=1$. (The uncertainty bands bracketing our results are explained following Eq. (31))

whereas the GRS DF yields 0.56 . We find once again that with our prediction $u^{\pi}\left(x ; \zeta_{0}^{\mathrm{LO}}\right) / u_{1}^{\pi}\left(x ; \zeta_{0}^{\mathrm{LO}}\right)>0.5$ is only realised on $x>0.99$.

Here and hereafter, following Refs. [39,40], we report results with an uncertainty determined by varying $\zeta_{H} \rightarrow$ $(1 \pm 0.1) \zeta_{H}$. Given that the precision of the infrared value of the PI coupling is $4 \%$, the $10 \%$ variation in $\zeta_{H}$ produces a conservative uncertainty estimate.

Beginning with Eq. (29), we generate glue and sea distributions from their initial identically-zero inputs using singlet evolution equations developed in analogy with that for nonsinglet evolution described in Sect.4.1. Our predictions at $\zeta=\zeta_{0}^{\mathrm{LO}}$ are displayed in Fig. $3 \mathrm{~b}$ and compared therein with the GRS input distributions at this scale. Not unexpectedly, our results do not support the use of valence-like distributions for glue and sea at this scale. An explicit comparison of the momentum fractions is nevertheless revealing:

\begin{tabular}{l|cc} 
& $\left\langle x g\left(x ; \zeta_{0}^{\mathrm{LO}}\right)\right\rangle\left\langle x S\left(x ; \zeta_{0}^{\mathrm{LO}}\right)\right\rangle$ \\
\hline herein & $0.24(5)$ & $0.03(1)$ \\
GRS & 0.29 & 0.15
\end{tabular}
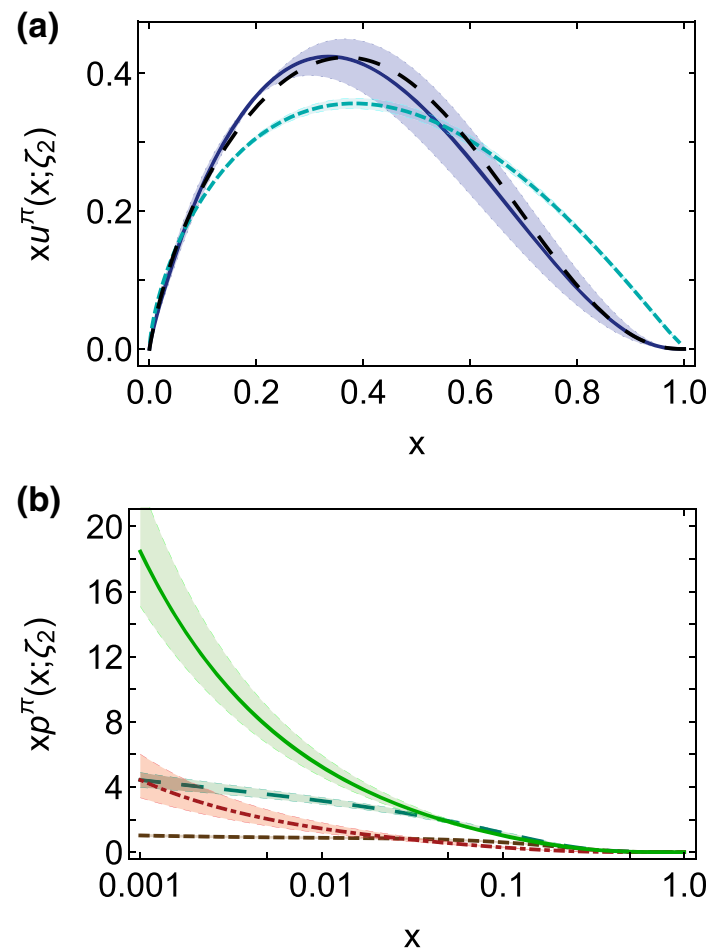

Fig. 4 a Solid blue curve - valence-quark distribution in Eq. (29) evolved to $\zeta=\zeta_{2}$, using the procedure explained in Sect.4.1; longdashed black curve - result from Ref. [102]; and short-dashed cyan - phenomenological result from Ref. [52], also at $\zeta=\zeta_{2}$. b Solid green curve, $p=g$ - our prediction for the pion's glue distribution; and dot-dashed red curve, $p=S$ - predicted sea-quark distribution. Phenomenological results from Ref. [52] are plotted for comparison: $p=$ glue - long-dashed dark-green; and $p=$ sea - dashed brown. Normalisation convention: $\left\langle x\left[2 u^{\pi}\left(x ; \zeta_{2}\right)+g^{\pi}\left(x ; \zeta_{2}\right)+S^{\pi}\left(x ; \zeta_{2}\right)\right]\right\rangle=1$. Notably, $2 u^{\pi}\left(x ; \zeta_{2}\right)>\left[g^{\pi}\left(x ; \zeta_{2}\right)+S^{\pi}\left(x ; \zeta_{2}\right)\right]$ on $x>0.2$, marking this as the valence domain within the pion. (The uncertainty bands bracketing our results are explained in the text)

The gluon momentum fractions are roughly comparable, but the sea contribution to the pion's momentum expressed in the GRS Ansatz is five-times greater than our prediction.

The models built in Refs. [101,103] were constrained by the GRS values for the glue and sea momentum fractions; hence, very likely placed too little momentum in the valence-quarks at $\zeta_{0}^{\mathrm{LO}}$. On the other hand, Ref. [102] estimated $\left\langle 2 x u^{\pi}\left(x ; \zeta_{0}^{\mathrm{LO}}\right)\right\rangle=0.71$, compatible with our prediction.

\subsection{2 $\zeta=\zeta_{2}=2 \mathrm{GeV}$}

Our prediction for $u^{\pi}\left(x ; \zeta_{2}\right)$ is depicted in Fig. $4 \mathrm{a}$. The solid curve and surrounding bands are described by the following function, a generalisation of Eq. (26):

$$
\begin{aligned}
& u^{\pi}(x)=\eta_{u^{\pi}} x^{\alpha}(1-x)^{\beta} \\
& \times\left[1+\rho x^{\alpha_{1} / 4}(1-x)^{\beta_{1} / 4}+\gamma x^{\alpha_{1} / 2}(1-x)^{\beta_{1} / 2}\right],
\end{aligned}
$$


Table 1 Coefficients and powers that reproduce the computed pion valence-quark distribution functions, depicted in Figs. 4, 5, when used in Eq. (33)

\begin{tabular}{cllllllr}
\hline & $n_{q^{\pi}}$ & $\alpha$ & $\beta$ & $\alpha_{1}$ & $\beta_{1}$ & $\rho$ \\
\hline$\zeta_{2}$ & 151 & 0.202 & 2.99 & 0.172 & 0.932 & -1.98 \\
& 132 & 0.112 & 3.11 & 0.145 & 0.917 & -1.95 \\
$\zeta_{5}$ & 112 & 0.0188 & 3.25 & 0.126 & 0.908 & -1.92 & 0.972 \\
& 116 & 0.0374 & 3.22 & 0.128 & 0.904 & -1.93 & 0.946 \\
& 101 & -0.0233 & 3.34 & 0.120 & 0.906 & -1.91 \\
\\
\hline
\end{tabular}

where $\eta_{u^{\pi}}$ ensures Eq. (25) and the powers and coefficients are listed in Table 1. These interpolations express the large- $x$ behaviour prescribed by Eq. (21); yet here $u^{\pi}\left(x ; \zeta_{2}\right) / u_{1}^{\pi}\left(x ; \zeta_{2}\right)>0.5$ is only realised on $x>0.99$. Plainly, subleading corrections to Eq. (21) play an important rôle at empirically accessible values of $x$. We therefore extract an "effective large- $x$ exponent", $\beta_{\text {eff }}(\zeta)$ by plotting $\ln u^{\pi}(x ; \zeta)$ against $\ln (1-x)$ on $x \in[0.9,1.0]$ and computing the slope, with the result

$\beta_{\text {eff }}\left(\zeta_{2}\right)=2.63(8)$

The uncertainty expresses that ascribed to $\zeta_{H}$. Notably, expanding the fitting domain to $x \in[0.85,1.0]$ and employing a jackknife analysis to obtain an array of slopes changes neither the central value nor the uncertainty at the quoted level of accuracy. For comparison, Refs. [39,40], which did not implement Eq. (21), determined $\beta\left(\zeta_{2}\right)=2.38(9)$ after evolution of $q_{\mathrm{O}}^{\pi}\left(x ; \zeta_{H}\right)$ in Eq. (26). It is worth remarking that Ref. [102] found $\beta\left(\zeta_{2}\right)=2.43$ and made no effort at an uncertainty estimate.

Here it is also worth providing a comparison of our calculated low-order moments with those obtained in some recent lattice QCD (1QCD) simulations $\left(\zeta=\zeta_{2}\right)$ :

\begin{tabular}{l|lll} 
& $\langle x\rangle_{u}^{\pi}$ & $\left\langle x^{2}\right\rangle_{u}^{\pi}$ & $\left\langle x^{3}\right\rangle_{u}^{\pi}$ \\
\hline lQCD [53] & $0.21(1)$ & $0.16(3)$ & \\
lQCD [54] & $0.254(03)$ & $0.094(12)$ & $0.057(04)$ \\
\hline Ref. [102] & 0.24 & 0.098 & 0.049 \\
Refs. [39, 40] & $0.24(2)$ & $0.098(10)$ & $0.049(07)$ \\
\hline Herein & $0.24(2)$ & $0.094(13)$ & $0.047(08)$
\end{tabular}

(We have simplified the notation: $\left\langle x^{n}\right\rangle_{u}^{\pi}=\left\langle x^{n} u^{\pi}(x)\right\rangle$, with the scale specified separately.) Plainly, continuum and 1QCD results agree on the light-front momentum fraction carried by valence-quarks in the pion at $\zeta=\zeta_{2}$ :

$\left\langle 2 x u^{\pi}\left(x ; \zeta_{2}\right)\right\rangle=0.47(2)$.

(Ref. [19, Table III] provides a larger array of comparisons, including model results.)

A similar valence-quark momentum fraction was obtained in Ref. [52] by analysing data on $\pi$-nucleus Drell-Yan (DY) and leading neutron electroproduction [52]: $\langle 2 x\rangle_{q}^{\pi}=0.49(1)$ at $\zeta=\zeta_{2}$. The associated phenomenological distribution is drawn as the short-dashed cyan curve in Fig.4a. Even though this DF yields a compatible momentum fraction, its $x$-profile is different. In fact, the phenomenological DF conflicts with the QCD constraint (Eq. 20). Significantly, the Ref. [52] analysis ignored threshold resummation effects, which are known to have a material impact at large $x[50,104]$. (Similar remarks apply to the analysis in Ref. [105].)

In our approach, as highlighted in the paragraph preceding that containing Eq. (3), a hadron's glue and sea distributions are identically zero at $\zeta_{H}$ : they are generated by evolution on $\zeta>\zeta_{H}$. Employing the same procedure used in developing our comparison with the GRS distributions, Eq. (32), we obtained the $\zeta=\zeta_{2}$ glue and sea distributions depicted in Fig. 4b. Adopting functional forms from Ref. [51], viz.

$x p(x)=\mathcal{A} x^{\alpha}(1-x)^{\beta}\left[1+\rho x^{1 / 2}+\gamma x\right]$,

$p=g, S$, these distributions are effectively interpolated using the coefficients in Table 2. The associated momentum fractions are $\left(\zeta=\zeta_{2}\right)$ :

$\langle x\rangle_{g}^{\pi}=0.41(2), \quad\langle x\rangle_{\text {sea }}^{\pi}=0.11(2)$.

References $[39,40]$ obtained 0.41(2), 0.11(2), respectively, from $q_{O}^{\pi}\left(x ; \zeta_{H}\right)$ in Eq. (26). (Although the model in Ref. [19] produces a pion valence-quark DF that disagrees with Eq. (20), it yields momentum fractions at $\zeta_{2}$ that are consistent with our predictions, viz. [106]: $\langle 2 x\rangle_{u}^{\pi}=0.49$, $\langle x\rangle_{g}^{\pi}=0.40,\langle x\rangle_{\mathrm{sea}}^{\pi}=0.11$.)

It is worth remarking that the ordering of our predictions agrees with that in Ref. [52]: $0.35(3), 0.16(1)$, but our gluon fraction is $\sim 20 \%$ larger and the sea fraction is $\sim 30 \%$ smaller. The associated DFs from Ref. [52] are drawn, respectively, as the long-dashed dark-green and shortdashed brown curves in Fig. 4b. Regarding the glue DFs, our prediction and the phenomenological result agree semiquantitatively on $x \gtrsim 0.05$; but they are markedly different on the complementary domain. Moreover, both glue DFs in Fig. 4 disagree with those inferred in earlier analyses [51,107]. These observations highlight the need for new experiments 
Table 2 Coefficients and powers that reproduce the computed pion's glue and sea distribution functions, depicted in Figs. 4, 5, when used in Eq. (37)

\begin{tabular}{|c|c|c|c|c|c|}
\hline & $\mathcal{A}$ & $\alpha$ & $\beta$ & $\rho$ & $\gamma$ \\
\hline \multirow[t]{3}{*}{$\zeta_{2}, g$} & 0.512 & -0.491 & 3.99 & -0.241 & 0.229 \\
\hline & 0.437 & -0.540 & 4.11 & 0.521 & -1.36 \\
\hline & 0.323 & -0.607 & 4.25 & 2.30 & -5.08 \\
\hline \multirow[t]{3}{*}{$\zeta_{2}, S$} & 0.141 & -0.455 & 4.99 & 0.958 & -2.36 \\
\hline & 0.127 & -0.506 & 5.11 & 2.19 & -4.82 \\
\hline & 0.111 & -0.564 & 5.25 & 3.52 & -7.50 \\
\hline \multirow[t]{3}{*}{$\zeta_{5}, g$} & 0.427 & -0.572 & 4.22 & -0.0932 & -0.229 \\
\hline & 0.340 & -0.622 & 4.34 & 0.856 & -1.36 \\
\hline & 0.271 & -0.676 & 4.48 & 1.56 & -5.08 \\
\hline \multirow[t]{3}{*}{$\zeta_{5}, S$} & 0.144 & -0.530 & 5.22 & 0.987 & -2.36 \\
\hline & 0.120 & -0.581 & 5.34 & 2.44 & -4.82 \\
\hline & 0.100 & -0.637 & 5.48 & 3.60 & -7.50 \\
\hline
\end{tabular}

that are directly sensitive to the pion's gluon content. This might be addressed through measurements of prompt photon and $J / \Psi$ production $[108,109]$.

The sea DFs in Fig. 4 have markedly different profiles on the entire $x$-domain. Hence, if the pion's gluon content is considered uncertain, then it is fair to describe the sea-quark distribution as empirically unknown. This is good motivation for the collection and analysis of DY data with $\pi^{ \pm}$beams on isoscalar targets $[108,110]$.

\section{$5.3 .3 \zeta=\zeta_{5}=5.2 \mathrm{GeV}$}

Drell-Yan data on the pion's valence-quark DF was taken in the E615 experiment [111]. Although this is the most recent set, it is more than thirty years old. Our predictions for the pion DFs at a scale appropriate for the E615 experiment, i.e. $\zeta_{5}=5.2 \mathrm{GeV}[49,111]$, are depicted in Fig. 5. The solid blue curve and surrounding bands are described by the function in Eq. (33) with the powers and coefficients listed in Table 1. Again, these interpolations express the large- $x$ behaviour prescribed by Eq. (21), but $u^{\pi}\left(x ; \zeta_{2}\right) / u_{1}^{\pi}\left(x ; \zeta_{2}\right)>0.5$ is only realised on $x>0.99$. Here,

$\beta_{\mathrm{eff}}\left(\zeta_{5}\right)=2.81(8)$,

a result consistent with Refs. [39,40]: $\beta\left(\zeta_{5}\right)=2.66(12)$.

Working with the $1 \mathrm{QCD}$ results obtained in Ref. [55], one finds $\beta_{\mathrm{IQCD}}\left(\zeta_{5}\right)=2.45(58)$; and also the following comparison between low-order moments:

\begin{tabular}{l|lll}
$\zeta_{5}$ & $\langle x\rangle_{u}^{\pi}$ & $\left\langle x^{2}\right\rangle_{u}^{\pi}$ & $\left\langle x^{3}\right\rangle_{u}^{\pi}$ \\
\hline Ref. [55] & $0.18(3)$ & $0.064(10)$ & $0.030(5)$ \\
Herein & $0.20(2)$ & $0.074(10)$ & $0.035(6)$
\end{tabular}
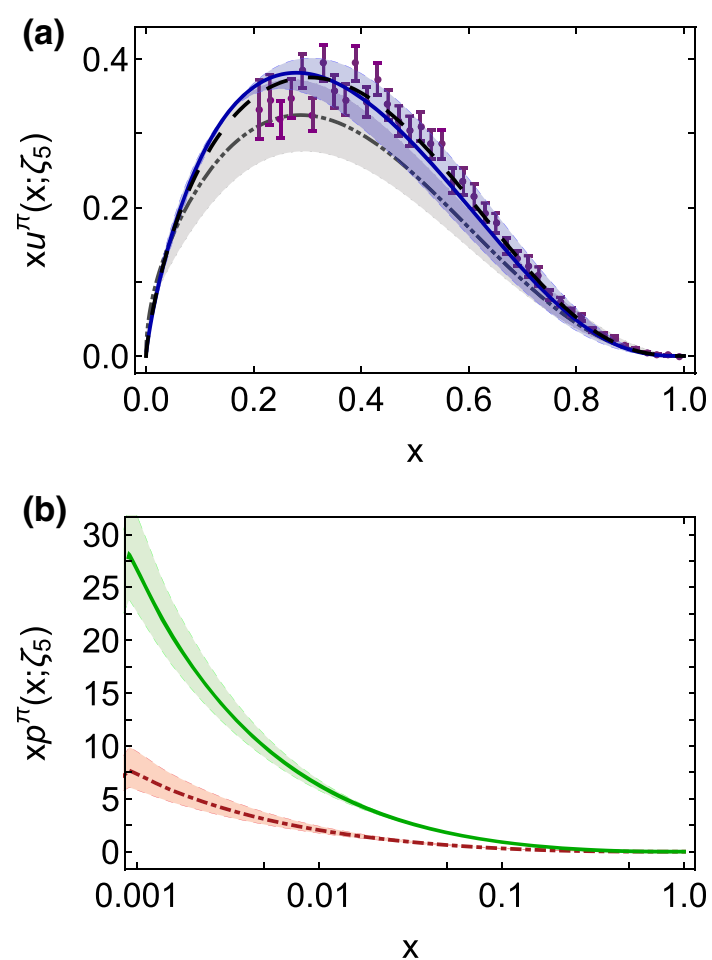

Fig. 5 a Solid blue curve - valence-quark distribution in Eq. (29) evolved to $\zeta=\zeta_{5}=5.2 \mathrm{GeV}$, using the procedure explained in Sect. 4.1; and long-dashed black curve - result from Ref. [102] at this scale. Dot-dot-dashed (grey) curve within shaded band - IQCD result [55]. Data (purple) from Ref. [111], rescaled according to the analysis in Ref. [50]. Comparing our central prediction with the plotted data, one obtains $\chi^{2} /$ d.o.f. $=1.66$. b Solid green curve, $p=g-$ our prediction for the pion's glue distribution; and dot-dashed red curve, $p=S$ - predicted sea-quark distribution. Normalisation convention: $\left\langle x\left[2 u^{\pi}\left(x ; \zeta_{5}\right)+g^{\pi}\left(x ; \zeta_{5}\right)+S^{\pi}\left(x ; \zeta_{5}\right)\right]\right\rangle=1$. (The uncertainty bands bracketing our results are explained in the text) 
Ref. [102] predicted, respectively: 0.21, 0.076, 0.036. We find

$\left\langle 2 x u^{\pi}\left(x ; \zeta_{5}\right)\right\rangle=0.41(4)$,

i.e. only $40 \%$ of the pion's momentum is carried by valence quarks at the E615 scale.

The data plotted in Fig. 5 is that reported in Ref. [111], rescaled according to the analysis in Ref. [50], which is NLO and includes soft-gluon resummation. Our prediction agrees with the rescaled data. No parameters were varied in order to achieve this outcome. Moreover, the EHMinduced broadening of the pion DF described in Sect.5.2 is crucial to the agreement.

The 1QCD result for the pion valence-quark DF [55] evolved to the E615 scale is drawn in Fig. 5 as the dot-dotdashed (grey) curve bracketed by grey bands: within errors, it agrees with our prediction. This is significant $[39,40]$ : two disparate treatments of the pion bound-state problem, one using continuum methods and the other using $1 \mathrm{QCD}$, have arrived at consistent results for the pion's valence-quark DF.

Our predictions for the pion's glue and sea DFs at $\zeta_{5}$ are displayed in Fig. 5b. From these DFs one obtains the following momentum fractions $\left(\zeta=\zeta_{5}\right)$ :

$\langle x\rangle_{g}^{\pi}=0.45(2), \quad\langle x\rangle_{\text {sea }}^{\pi}=0.14(2)$,

in agreement with Refs. $[39,40]$. The DFs are described by the function in Eq. (37) evaluated using the appropriate coefficients and powers in Table 2.

To highlight the importance of a complete next-to-leadingorder treatment, which includes threshold resummation effects, in any analysis of data whose aim is extraction of a valence-quark DF, Fig. 6 compares our prediction for $u^{\pi}\left(x ; \zeta_{5}\right)$ and that from Ref. [55] with the results published in Ref. [111]. The latter were obtained in a straightforward LO analysis; and disagree markedly with modern predictions on $x \gtrsim 0.5$.

In closing this section it is worth recalling another textbook result. Namely, on $\Lambda_{\mathrm{QCD}}^{2} / \zeta^{2} \simeq 0$, for any hadron [112]: $\langle x\rangle_{q}=0,\langle x\rangle_{g}=4 / 7 \approx 0.57,\langle x\rangle_{S}=3 / 7 \approx 0.43$. This means there is a scale beyond which DFs cannot provide information that enables distinctions to be drawn between different hadrons: for each one, the valence distribution is a $\delta$-function located at $x=0[113-115]$.

\section{Kaon distribution amplitude}

Calculations of the kaon's leading twist DA, $\varphi_{K}(x)$, using Eq. (6b) are reported in Refs. [74,75]. They are complemented by related analyses of the kaon electromagnetic elastic form factor $[65,77]$ and $1 Q C D$ results for the DA's first two nontrivial moments [116-118]. These analyses reveal that whilst the pion's DA is well constrained, the kaon's is

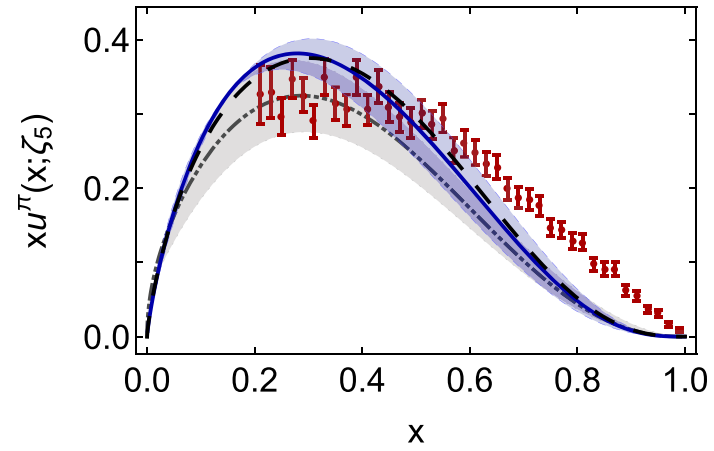

Fig. 6 Solid blue curve - valence-quark distribution in Eq.(29) evolved to $\zeta=\zeta_{5}=5.2 \mathrm{GeV}$, using the procedure explained in Sect. 4.1; long-dashed black curve - result from Ref. [102] at this scale; and dot-dot-dashed (grey) curve within shaded band-1QCD result [55]. Data (red) - DF as determined in a straightforward LO analysis [111]. Comparing our central prediction with the data plotted here, one obtains $\chi^{2} /$ d.o.f. $=19.4$. (The uncertainty bands bracketing our results are explained in the text)

more uncertain. One can at most conclude the following: (i) $\varphi_{K}\left(x ; \zeta_{H}\right)$ is somewhat less broadened than $\varphi_{\pi}\left(x ; \zeta_{H}\right)$; and (ii) it is slightly asymmetric about $x=1 / 2$ owing to the significantly smaller role played by the Higgs mechanism of mass generation for $u$-quarks as contrasted with $s$-quarks, viz. $M_{u}\left(k^{2}=0\right) / M_{S}\left(k^{2}=0\right) \approx f_{\pi} / f_{K} \approx 0.84$. Defining

$\left\langle\xi^{n}=(1-2 x)^{n}\right\rangle_{M}^{u_{\zeta}}=\int_{0}^{1} d x(1-2 x)^{n} \varphi_{M}^{u}(x ; \zeta)$,

these remarks can be stated quantitatively as follows:

$$
\begin{aligned}
\left\langle\left[\xi, \xi^{2}\right]\right\rangle_{\pi}^{u_{\zeta_{H}}} & =[0,0.25], \\
\left\langle\left[\xi, \xi^{2}\right]\right\rangle_{K}^{u_{\zeta_{H}}} & =[0.035(5), 0.24(1)] .
\end{aligned}
$$

Following the procedures described in Ref. [74] and Sect. 5.2 herein, Eq. (44b) can be used to determine the following pointwise form for the kaon's DA:

$$
\begin{aligned}
& \varphi_{K}^{u}\left(x ; \zeta_{H}\right)=n_{\varphi_{K}} x(1-x) \\
& \quad \times\left[1+\rho x^{\frac{\alpha}{2}}(1-x)^{\frac{\beta}{2}}+\gamma x^{\alpha}(1-x)^{\beta}\right],
\end{aligned}
$$

where $n_{\varphi_{K}}$ ensures unit normalisation, and the interpolation coefficients are listed in Table 3. Here "upper" indicates the curve that produces the largest value of $\left\langle\xi^{2}\right\rangle_{K}^{u_{\zeta H}}$ and lower, the smallest. $\varphi_{K}^{\bar{s}}\left(x ; \zeta_{H}\right)$ is obtained using Eq. (8).

The DA family described by Eq. (45) and the coefficients in Table 3 is drawn in Fig. 7 as the solid blue curve within blue shading. For comparison, the dot-dashed red curve is the kaon DA obtained using the DB kernel in Ref. [74]. The agreement between our curves and that result is good; especially since no uncertainty estimate was provided in Ref. [74], yet that uncertainty cannot be entirely negligible because numerous 
Table 3 Coefficients and powers that specify the kaon DA determined by Eq. (45). Upper, middle, lower refer to the values of $\left\langle\xi^{2}\right\rangle_{K}^{u_{\zeta H}}$ produced by the identified coefficients. The "upper" parameter values produce the curve in Fig. 7 with the smallest magnitude at $x=0.5$, etc

\begin{tabular}{llllll}
\hline & $n_{\varphi_{K}}$ & $\rho$ & $\gamma$ & $\alpha$ & $\beta$ \\
\hline Upper & 16.2 & 4.92 & -6.00 & 0.0946 & 0.0731 \\
Middle & 18.2 & 5.00 & -5.97 & 0.0638 & 0.0481 \\
Lower & 20.2 & 5.00 & -5.90 & 0.0425 & 0.0308 \\
\hline
\end{tabular}

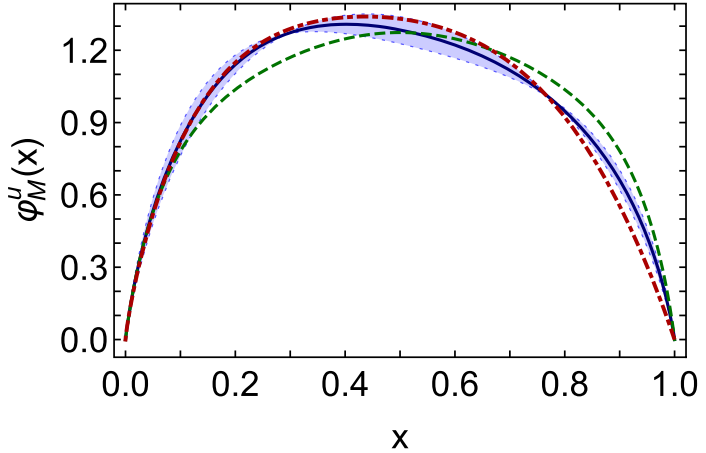

Fig. 7 Kaon DA, $\varphi_{K}^{u}\left(x ; \zeta_{H}\right)$, described by Eq. (45) and the "middle" coefficients in Table 3 - solid blue curve. The associated band marks the domain bounded by the "upper" and "lower" coefficients in Table 3. Result obtained using the DB kernel in Ref. [74] - dot-dashed red curve. Pion DA in Eq. (28) - dashed green curve

interpolations and extrapolations of propagators and amplitudes were employed to complete the calculation. The level of agreement is further highlighted by a comparison between moments:

\begin{tabular}{c|cccc} 
& $\langle\xi\rangle_{K}^{u_{\zeta_{H}}}$ & $\left\langle\xi^{2}\right\rangle_{K}^{u_{\zeta_{H}}}$ & $\left\langle\xi^{3}\right\rangle_{K}^{u_{\zeta_{H}}}$ & $\left\langle\xi^{4}\right\rangle_{K}^{u_{\zeta_{H}}}$ \\
\hline Ref. [74] & 0.040 & 0.23 & 0.021 & 0.11 \\
herein & $0.035(5)$ & $0.24(1)$ & $0.016(2)$ & $0.11(1)$
\end{tabular}.

In order to provide immediately for a contrast between pion and kaon DAs, the pion DA in Eq. (28) is drawn as the dashed green curve in Fig. 7.

\section{Kaon distribution functions}

\subsection{Hadron scale}

Having established that the results for the kaon's DA are sound, we identify Eq. (12) as our prediction for the kaon's valence-quark DFs; viz., using the "middle" curve as representative:

$$
\begin{aligned}
u^{K}\left(x ; \zeta_{H}\right)= & 299.18 x^{2}(1-x)^{2}[1+5.00 \\
& \left.\times x^{0.032}(1-x)^{0.024}-5.97 x^{0.064}(1-x)^{0.048}\right]^{2},
\end{aligned}
$$

with $\bar{s}^{K}\left(x ; \zeta_{H}\right)=u^{K}\left(1-x ; \zeta_{H}\right)$.

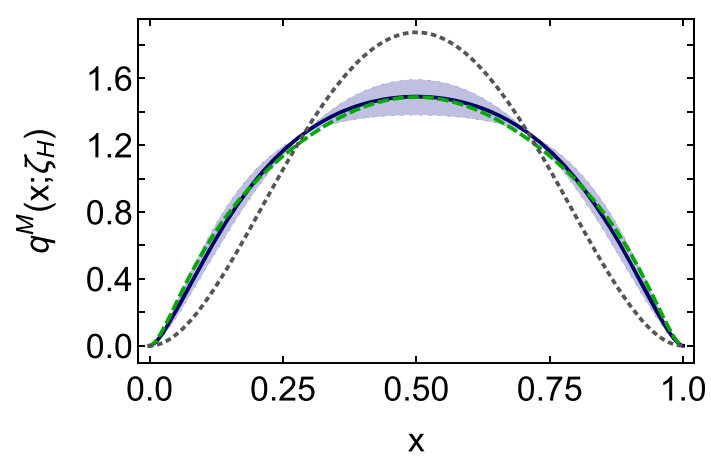

Fig. 8 A comparison between kaon and pion valence-quark DFs. Solid blue curve: $\frac{1}{2}\left[u^{K}\left(x ; \zeta_{H}\right)+\bar{s}^{K}\left(x ; \zeta_{H}\right)\right]$ calculated from Eq. (47). The associated band marks the domain bounded by the kaon DFs produced using Eqs. (12), (45) and the "upper" and "lower" rows in Table 3. Dashed green curve: $u^{\pi}\left(x ; \zeta_{H}\right)$ in Eq. (29). Dotted grey curve: scale free form, $q^{\text {sf }}\left(x ; \zeta_{H}\right)=30 x^{2}(1-x)^{2}$

An instructive comparison between the kaon's valencequark DFs and that of the pion is depicted in Fig. 8, viz. $\frac{1}{2}\left[u^{K}\left(x ; \zeta_{H}\right)+\bar{s}^{K}\left(x ; \zeta_{H}\right)\right]$ vs. $u^{\pi}\left(x ; \zeta_{H}\right)$. The evident similarity between these curves is determined by Eqs. (10), (12), (44). Namely: (i) at $\zeta_{H}$, the dressed-valence quasiparticles express all properties of the bound state under consideration; and (ii) relative to the pion, the impact of EHM, as expressed in broadening of parton distributions, is only marginally less strong in the kaon. The broadening distinguishes physical light- and lighter-quark DFs from the scale free form: $q^{\text {sf }}\left(x ; \zeta_{H}\right)=30 x^{2}(1-x)^{2}$.

At this point, one more independent check is possible. Namely, working with the DB-kernel propagators and amplitudes reported in Ref. [74], Eq. (22) can be used directly to calculate the lowest four nontrivial moments of the kaon DF.

\begin{tabular}{|c|c|c|c|}
\hline$\zeta_{H}$ & $\left\langle x^{2} u^{K}\right\rangle$ & $\left\langle x^{3} u^{K}\right\rangle$ & $\left\langle x^{4} u^{K}\right\rangle$ \\
\hline Eq. $(22)^{[74]}$ & $0.456(4) 0$ & 146 & 3( \\
\hline Eq. (47) & 5) 0.2 & $73(1)$ & $0.120(2)$ \\
\hline
\end{tabular}
This procedure yields the following comparison:

This is a favourable comparison, especially since the DA in Ref. [74] is similar to ours but not identical, being less dilated - see Fig. 7 and Eq. (46). Owing to Eq. (10),

$\left\langle x \bar{s}^{K}\right\rangle=1-\left\langle x u^{K}\right\rangle=0.529(5)$. 


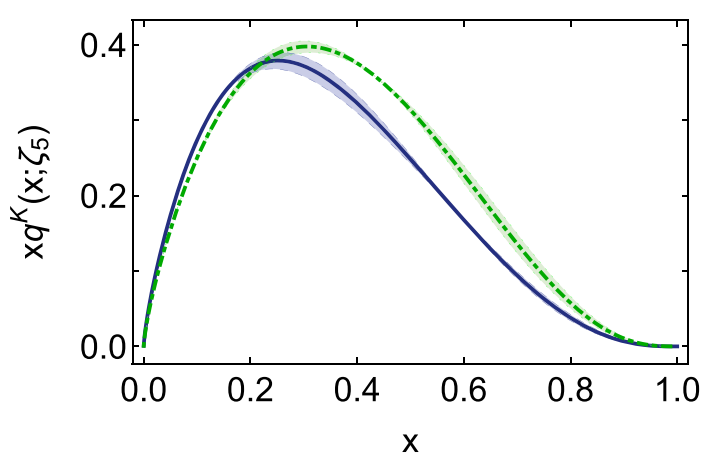

Fig. 9 Kaon DFs, defined at $\zeta_{H}$ by Eqs. (12), (45) and Table 3, evolved $\zeta_{H} \rightarrow \zeta_{5}$ : solid blue curve $-q=u$; and dot-dashed green curve $-q=\bar{s}$. The like-coloured bands surrounding each curve mark the domain between the "upper" and "lower" results from Table 3: "lower" produces the curve with greatest magnitude in the peak region and smallest magnitude at large $x$

Table 4 Coefficients and powers that provide interpolations for the computed kaon valence-quark distribution functions, depicted in Fig. 9, when used in Eq. (33): Rows 1-3-u-quark; and Rows 4-6 - $\bar{s}$-quark

\begin{tabular}{llllllll}
\hline$u^{K}$ & $n_{u^{K}}$ & $\alpha$ & $\beta$ & $\alpha_{1}$ & $\beta_{1}$ & $\rho$ & $\gamma$ \\
\hline$\zeta_{5}$ & 40.8 & -0.0371 & 3.34 & 0.319 & 1.82 & -2.15 & 1.26 \\
& 50.9 & 0.0508 & 3.34 & 0.363 & 1.46 & -2.11 & 1.20 \\
& 61.8 & -0.190 & 3.34 & 0.000 & 0.533 & -1.54 & 0.578 \\
\hline $\bar{s}^{K}$ & $n_{\bar{s}^{K}}$ & $\alpha$ & $\beta$ & $\alpha_{1}$ & $\beta_{1}$ & $\rho$ & $\gamma$ \\
\hline$\zeta_{5}$ & 40.8 & 0.0812 & 3.34 & 0.483 & 2.70 & -2.23 & 1.36 \\
& 50.9 & -0.0251 & 3.34 & 0.168 & 1.63 & -1.86 & 0.902 \\
& 61.8 & -0.205 & 3.34 & 0.0011 & 1.02 & -1.70 & 0.727 \\
\hline
\end{tabular}

(The uncertainty estimate in Row 1 of Eq. (48) is based on a $\pm 30 \%$ variation in the strength of the skewing-term in the DB-kernel result for the Bethe-Salpeter amplitude. Such variation changes the calculated value of $f_{K}=0.11 \mathrm{GeV}$ by $<0.2 \%$.)

\subsection{Evolved kaon DFs: massless splitting}

Regarding kaon structure functions, the only available empirical information is the ratio $u^{K}(x) / u^{\pi}(x)$, which was measured in the production of massive muon pairs forty years ago [119]. The mass-scale in this experiment is similar to that of E615, i.e. $\zeta \approx \zeta_{5}$. Thus, in order to deliver results for comparison with this DY data, our kaon valence-quark DFs must be evolved: $\zeta_{H} \rightarrow \zeta_{5}$.

It is straightforward to employ the approach explained in Sect. 4.1, used above for the pion, to evolve the kaon DFs defined at $\zeta_{H}$ by Eqs. (12), (45) and Table 3 to the E615 scale, $\zeta_{5}$. This procedure yields the curves plotted in Fig. 9, which may be interpolated using the functional form in Eq. (33) and the coefficients and powers in Table 4. The curves in Fig.9 produce the following low-order moments:

$$
\begin{array}{l|c|c|c}
q \backslash \zeta_{5} & \left\langle x q^{K}\right\rangle & \left\langle x^{2} q^{K}\right\rangle & \left\langle x^{3} q^{K}\right\rangle \\
\hline u & 0.193(\mp 2) & 0.0669(\mp 04) & 0.0301( \pm 01) \\
\bar{s} & 0.217( \pm 2) & 0.0812( \pm 22) & 0.0386( \pm 17)
\end{array}
$$

Combining the results in column 1 , one finds

$$
\left\langle x\left[u^{K}\left(x ; \zeta_{5}\right)+\bar{s}^{K}\left(x ; \zeta_{5}\right)\right]\right\rangle=0.410 ;
$$

hence, using mass-independent splitting functions, the valencequark momentum fraction in the kaon at $\zeta_{5}$ is the same as that in the pion (Eq. 41). (The uncertainties listed here derive from the variation in kaon DAs, illustrated in Fig. 7.)

Using the $\zeta_{H} \rightarrow \zeta_{5}$ evolved kaon DFs, one obtains the ratio $u^{K}\left(x ; \zeta_{5}\right) / u^{\pi}\left(x ; \zeta_{5}\right)$ drawn in Fig. 10 . Evidently, the uncertainty existing in the kaon DA is expressed in the behaviour of this ratio on $x \gtrsim 0.5$ : a broader kaon DA yields a ratio closer to unity at $x=1$. The central curve is interpolated by the function:

$$
\begin{aligned}
& \frac{u^{K}\left(x ; \zeta_{5}\right)}{u^{\pi}\left(x ; \zeta_{5}\right)} \\
& \quad=\frac{1.04-2.54 x+3.87 x^{2}+3.82 x^{3}-3.90 x^{4}}{1-2.61 x+5.64 x^{2}} .
\end{aligned}
$$

The best agreement with extant data [119] is delivered by the kaon DF written in Eq. (47). Thus, hereafter, we focus on the kaon DFs defined by this curve; and, just as we did above for the pion, consider the impact of varying $\zeta_{H} \rightarrow(1.0 \pm 0.1) \zeta_{H}$, thereby providing a conservative estimate of the uncertainty arising from that in the infrared value of the PI coupling, Fig. 1. This process yields the DFs plotted in Fig. 11a, which can be interpolated using the functional form in Eq. (33) and the coefficients and powers in Table 5. These interpolations express the large- $x$ behaviour indicated in Eq. (21), but $q^{K}\left(x ; \zeta_{5}\right) / q_{1}^{K}\left(x ; \zeta_{5}\right)>0.5$ is only realised on $x>0.95$. Here, consistent with Eq. (39):

$\beta_{\text {eff }}\left(\zeta_{5}\right)=2.73(7)$.

The kaon DFs just described produce the following loworder moments:

\begin{tabular}{l|c|c|c}
$q \backslash \zeta_{5}$ & $\left\langle x q^{K}\right\rangle$ & $\left\langle x^{2} q^{K}\right\rangle$ & $\left\langle x^{3} q^{K}\right\rangle$ \\
\hline$u$ & $0.19(2)$ & $0.067(09)$ & $0.030(5)$ \\
$\bar{s}$ & $0.22(2)$ & $0.081(11)$ & $0.038(7)$
\end{tabular}

hence, accounting for $\zeta_{H} \rightarrow \zeta_{H}(1.0 \pm 0.1)$,

$\left\langle x\left[u^{K}\left(x ; \zeta_{5}\right)+\bar{s}^{K}\left(x ; \zeta_{5}\right)\right]\right\rangle=0.41(4)$,

reproducing the pion result (Eq. 41).

First $1 Q C D$ results for the kaon's valence-quark DFs are now available [120]. The study finds the following moments, listed here in the order of appearance in Eq.(54): $u-$ 0.193(8), 0.080(7), 0.042(6); and $\bar{s}-0.267(8), 0.123(7)$, $0.070(6)$. These values are systematically larger than our 


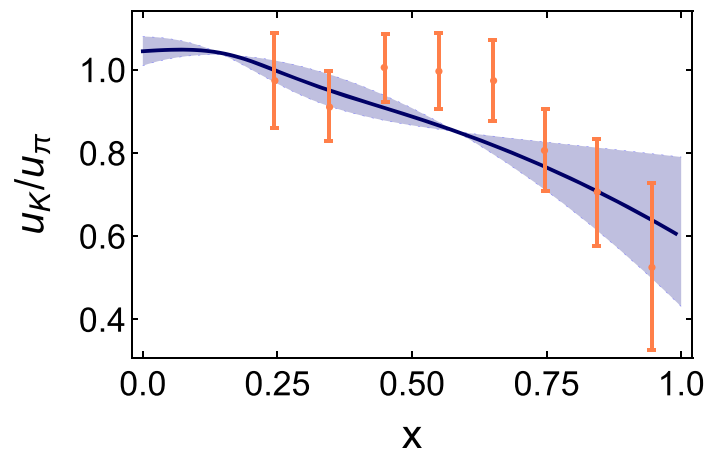

Fig. $10 u^{K}\left(x ; \zeta_{5}\right) / u^{\pi}\left(x ; \zeta_{5}\right)$. Solid blue curve - result obtained after evolution of Eq. (29) $[\pi]$ and Eq. (47) $[K]$. (Eq. (52) provides an interpolation for this curve.) The light-blue band marks the domain between the results obtained using Eqs. (12), (45), Table 3-upper and Eqs. (12), (45), Table 3-lower, with "lower" producing the smallest value at $x=1$. Data (orange) from Ref. [119]

Table 5 Coefficients and powers that provide interpolations for the computed kaon valence-quark distribution functions, depicted in Fig. 11, when used in Eq. (33): Rows 1-3 - $u$-quark; and Rows 4-6 $\bar{s}$-quark

\begin{tabular}{llllllll}
\hline$u^{K}$ & $n_{u^{K}}$ & $\alpha$ & $\beta$ & $\alpha_{1}$ & $\beta_{1}$ & $\rho$ & $\gamma$ \\
\hline$\zeta_{5}$ & 60.9 & 0.115 & 3.22 & 0.358 & 1.39 & -2.10 & 1.89 \\
& 50.9 & 0.0508 & 3.34 & 0.363 & 1.46 & -2.11 & 1.19 \\
& 40.6 & -0.0354 & 3.48 & 0.334 & 1.49 & -2.06 & 1.16 \\
\hline$\overline{\mathcal{s}}^{K}$ & $n_{\bar{s}^{K}}$ & $\alpha$ & $\beta$ & $\alpha_{1}$ & $\beta_{1}$ & $\rho$ & $\gamma$ \\
\hline$\zeta_{5}$ & 60.9 & 0.0399 & 3.22 & 0.180 & 1.58 & -1.89 & 0.930 \\
& 50.9 & -0.0251 & 3.34 & 0.168 & 1.63 & -1.86 & 0.902 \\
& 40.6 & -0.0900 & 3.48 & 0.165 & 1.71 & -1.83 & 0.879 \\
\hline
\end{tabular}

predictions, especially for the $\bar{s}$, viz. the excesses are: $u$ - 0.6(4.8)\%, 21(6)\%, 40(4)\%; and $\bar{s}-24(7) \%, 53(13) \%$, $84(16) \%$. This is because, when compared with our predictions, the 1QCD DFs are much harder; a feature highlighted by Fig. 11b. In fact, the 1QCD results are inconsistent with the QCD prediction in Eq. (20): on $x \simeq 1$, the lQCD DF behaves as $(1-x)^{\beta}, \beta=1.13(16)$. We expect that future refinements of IQCD setups, algorithms and analyses will move the lattice results closer to ours.

It is interesting that even though the valence-quark DFs computed using the model Hamiltonian in Ref. [19] are also harder than those in Fig. 11 and in conflict with the QCD constraint (Eq. 20), the pattern of comparison with the lQCD results [120] is similar. Namely, the valence-quark 1QCD moments, especially those associated with the $\bar{s}$, are significantly larger than those produced by the model.

Figure 12 depicts the ratio $u^{K}\left(x ; \zeta_{5}\right) / u^{\pi}\left(x ; \zeta_{5}\right)$ obtained by evolving the pion and kaon DFs $\zeta_{H} \rightarrow \zeta_{5}$, accounting for a $10 \%$ uncertainty in $\zeta_{H}$. Evidently, the impact of $\zeta_{H} \rightarrow \zeta_{H}(1.0 \pm 0.1)$ on both $u^{\pi}\left(x ; \zeta_{5}\right)$ and $u^{K}\left(x ; \zeta_{5}\right)$ is
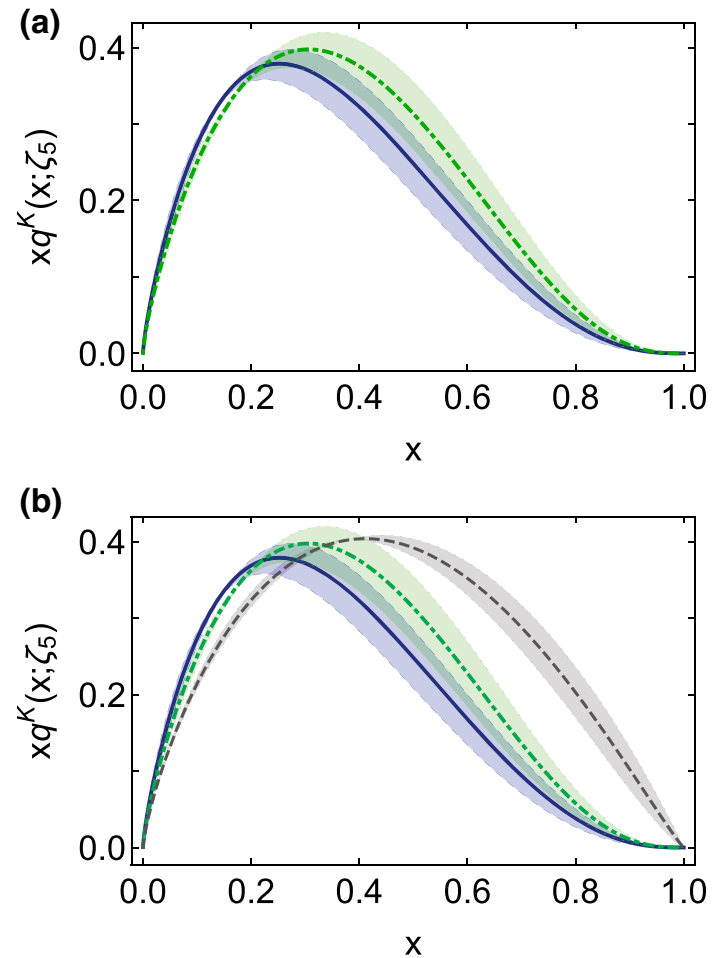

Fig. 11 a Solid blue curve - kaon's valence $u$-quark distribution, defined at $\zeta_{H}$ by Eq. (47), evolved $\zeta_{H} \rightarrow \zeta_{5}$ using the procedure explained in Sect. 4.1. Dot-dashed green curve - analogous result for the kaon's valence $\bar{s}$ distribution. b Dashed grey curve within grey bands - kaon $\bar{s}$ valence-quark distribution obtained in a recent IQCD study [120]; otherwise, as in A. (In both panels, the bands bracketing our central DF curves reflect the uncertainty in $\hat{\alpha}(0)$, Fig. 1)

almost identical because the ratio exhibits practically no sensitivity. It is worth highlighting here that Eqs. (17)-(21) guarantee that the large- $x$ power-law exponents of $u^{\pi}(x, \zeta)$ and $u^{\mathrm{K}}(x, \zeta)$ evolve at the same rate, in consequence of which the ratio $u^{\mathrm{K}}\left(x, \zeta_{5}\right) / u^{\pi}\left(x, \zeta_{5}\right)$ is nonzero and finite on $x \simeq 1$.

The first lQCD results for this ratio are also drawn in Fig. 12. The relative difference between the central $1 Q C D$ result and our prediction is $\approx 5 \%$ despite the fact that the individual 1QCD DFs are qualitatively and quantitatively different from ours, drawn in Figs. 5, 9, 11. This feature highlights a long known characteristic, i.e. $u^{K}\left(x ; \zeta_{5}\right) / u^{\pi}\left(x ; \zeta_{5}\right)$ is quite forgiving of even large differences between the individual DFs used to produce the ratio, as may be seen by comparing, e.g. Refs. [17,19,121-123]. More precise data is crucial if this ratio is to be used effectively to inform and test the modern understanding of SM NG modes; and results for $u^{\pi}\left(x ; \zeta_{5}\right), u^{K}\left(x ; \zeta_{5}\right)$ separately have greater discriminating power $[108,124,125]$.

\subsection{Evolved kaon DFs: mass-dependent splitting}

Hitherto, when implementing the evolution procedure described in Sect.4.1, we have used textbook forms of the massless 


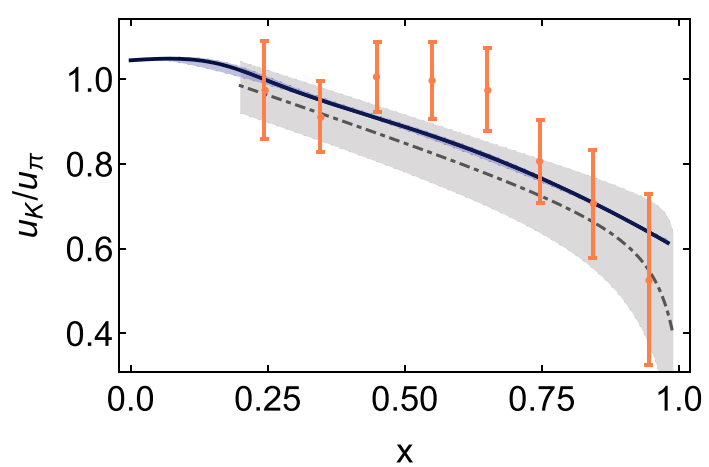

Fig. $12 u^{K}\left(x ; \zeta_{5}\right) / u^{\pi}\left(x ; \zeta_{5}\right)$. Solid blue curve - result obtained for the ratio after $\zeta_{H} \rightarrow \zeta_{5}$ evolution of Eq. (29) [ $[\pi]$ and Eq. (47) $[K]$. (Eq. (52) provides an interpolation for this curve.) The lighter-blue band bracketing the curve reveals the effect of $\zeta_{H} \rightarrow \zeta_{H}(1.0 \pm 0.1)$ : it is negligible. Dot-dashed grey curve within grey band-1QCD result [120]. Data (orange) from Ref. [119]

splitting functions. Consequently, given the results highlighted in Fig. 8, the glue and sea distributions in the kaon are practically identical to those in the pion. Indeed, any symmetry-preserving study that begins at $\zeta_{H}$ with a boundstate constituted solely from dressed quasiparticles and implements physical constraints on $\pi$ and $K$ wave functions will deliver this outcome when using massless splitting functions. Of course, the $s$-quark is more massive than the $u$-quark. Hence, physically [126,127]: valence $\bar{s}$-quarks must produce less gluons than valence $u$-quarks; and gluon splitting must produce less $\bar{s} s$-pairs than light-quark pairs. Such effects can be expressed in the splitting functions and we now illustrate their impact.

The integro-differential equations describing singlet evolution involve a splitting function kernel of the form

$$
\left(\begin{array}{cc}
P_{q \leftarrow q} & P_{q \leftarrow g} \\
P_{g \leftarrow q} & P_{g \leftarrow g}
\end{array}\right)
$$

where the $P_{a \leftarrow b}$ are splitting functions and the subscript indicates the direction of momentum transfer between participating partons. Momentum conservation imposes the following constraints:

$0=\int_{0}^{1} d z z\left[P_{q \leftarrow q}(z)+P_{g \leftarrow q}(z)\right]$,

$0=\int_{0}^{1} d z z\left[2 n_{f} P_{q \leftarrow g}(z)+P_{g \leftarrow g}(z)\right]$,

$P_{g \leftarrow q}(z)=P_{q \leftarrow q}(1-z), P_{q \leftarrow g}(z)=P_{q \leftarrow g}(1-z), P_{g \leftarrow g}(z)$ $=P_{g \leftarrow g}(1-z)$. Moreover, baryon number conservation requires that the complete evolution kernel guarantees

$0=\int_{0}^{1} d x \frac{d}{d \ln \zeta} q^{M}(x ; \zeta)$

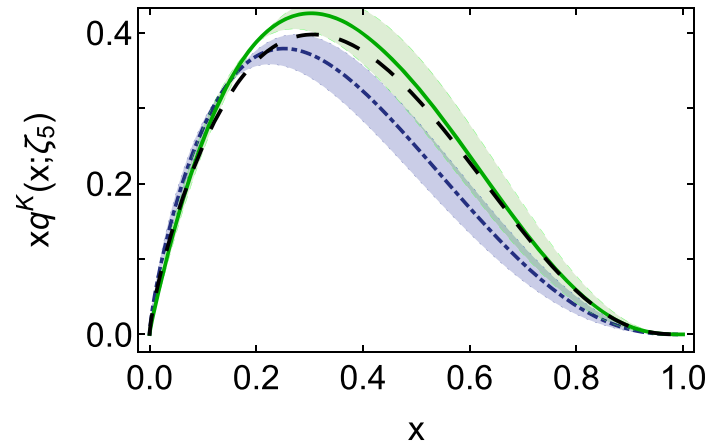

Fig. 13 Solid green curve-kaon's valence $\bar{s}$-quark distribution defined at $\zeta_{H}$ by Eqs. (10), (47), evolved $\zeta_{H} \rightarrow \zeta_{5}$ using the procedure explained in Sect.4.1, including the splitting function modification in Eq. (59a) with $\sigma_{s}=1$. Dot-dashed blue curve - kaon's valence $u$-quark distribution, unchanged from Fig. 11. Dashed black curve - central $\bar{s}$-quark distribution from Fig. 11, i.e. obtained with mass-independent evolution. (The bands bracketing the central DFs reflect the uncertainty in the $k^{2}=0$ value of the PI charge, Fig. 1 )

There are many prescriptions for introducing mass dependence into the splitting functions, e.g. Refs. [128-130]; and a novel formulation will be presented elsewhere [131]. Herein, however, we implement a simple expedient for the purpose of illustrating the typical outcome. Namely, we identify and separate the quark flavours, modify the $s$-quark evolution kernel contributions as follows:

$$
\begin{aligned}
& P_{s \leftarrow s}(z) \rightarrow P_{q \leftarrow q}(z)-\Delta_{s \leftarrow s}(z, \zeta), \\
& P_{s \leftarrow g}(z) \rightarrow P_{s \leftarrow g}(z)+\Delta_{s \leftarrow g}(z, \zeta),
\end{aligned}
$$

and use the following Ansätze for the modifications

$$
\begin{aligned}
& \Delta_{s \leftarrow s}(z, \zeta)=\sqrt{3}(1-2 z) \frac{\sigma_{S} \delta^{2}}{\delta^{2}+\left(\zeta-\zeta_{H}\right)^{2}}, \\
& \Delta_{s \leftarrow g}(z, \zeta)=\sqrt{5}\left(1-6 z+6 z^{2}\right) \frac{\sigma_{S} \delta^{2}}{\delta^{2}+\left(\zeta-\zeta_{H}\right)^{2}}
\end{aligned}
$$

which, following Ref. [101], are based on the simplest two nontrivial Gegenbauer polynomials that ensure the momentum conservation constraints, Eq. (57). Here, $\delta=0.1 \mathrm{GeV} \approx$ $M_{s}\left(k^{2}=0\right)-M_{u}\left(k^{2}=0\right) \approx \hat{m}_{s}-\hat{m}_{u}$ and $\sigma_{s}>0$ is a strength parameter.

The impacts of these modifications are clear: Eq. (59a) serves to reduce the number of gluons emitted by $\bar{s}$-quarks; and Eq. (59b) suppresses the density of $s \bar{s}$ pairs produced by gluons. Both effects increase with the quark mass difference, $\delta$, and decrease as $\delta^{2} / \zeta^{2}$ with increasing resolving scale.

We plot the new results for $\bar{s}^{K}\left(x ; \zeta_{5}\right)$ in Fig. 13. Obtained with $\sigma_{s}=1$, these DFs produce the following low-order 
Table 6 Coefficients and powers that provide interpolations for the computed kaon valence-quark distribution functions, depicted in Fig. 13, when used in Eq. (33): Rows 1-3-u-quark, unchanged from Table 5, included here to simplify comparisons; and Rows $4-6-\bar{s}$ quark, obtained using mass-dependent splitting functions, Eq. (59)

\begin{tabular}{llllllll}
\hline$u^{K}$ & $n_{u^{K}}$ & $\alpha$ & $\beta$ & $\alpha_{1}$ & $\beta_{1}$ & $\rho$ & $\gamma$ \\
\hline$\zeta_{5}$ & 60.9 & 0.115 & 3.22 & 0.358 & 1.39 & -2.10 & 1.89 \\
& 50.9 & 0.0508 & 3.34 & 0.363 & 1.46 & -2.11 & 1.19 \\
& 40.6 & -0.0354 & 3.48 & 0.334 & 1.49 & -2.06 & 1.16 \\
\hline $\bar{s}^{K}$ & $n_{\bar{s} K}$ & $\alpha$ & $\beta$ & $\alpha_{1}$ & $\beta_{1}$ & $\rho$ & $\gamma$ \\
\hline$\zeta_{5}$ & 66.6 & 0.168 & 3.16 & 0.217 & 1.42 & -1.89 & 0.947 \\
& 56.8 & 0.119 & 3.27 & 0.222 & 1.45 & -1.88 & 0.945 \\
& 46.5 & 0.0608 & 3.40 & 0.234 & 1.50 & -1.88 & 0.960 \\
\hline
\end{tabular}

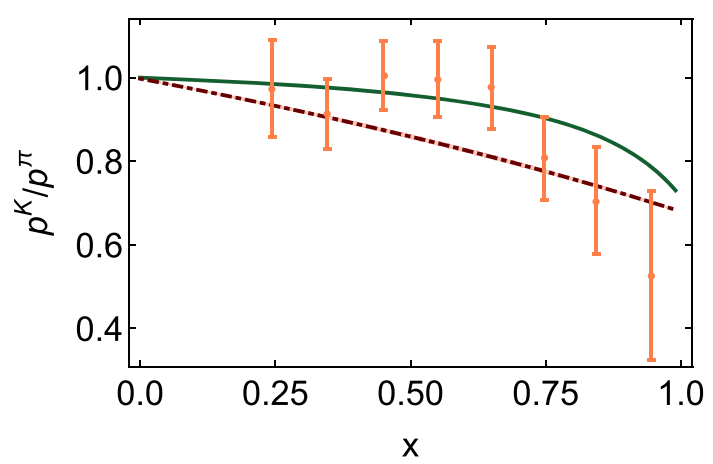

Fig. 14 Prediction for $g^{K}\left(x ; \zeta_{5}\right) / g^{\pi}\left(x ; \zeta_{5}\right)$ - solid green curve within green shading; and for $\mathcal{S}^{K}\left(x ; \zeta_{5}\right) / \mathcal{S}^{\pi}\left(x ; \zeta_{5}\right)$ - dot-dashed red curve within red shading. (The uncertainty introduced by that in the $k^{2}=0$ value of the PI charge, Fig. 1, is indicated by the shaded band bracketing each curve. In both cases here, that band is no thicker than the width of the central line.) Data on $u^{K}\left(x ; \zeta_{5}\right) / u^{\pi}\left(x ; \zeta_{5}\right)$ (orange) from Ref. [119] are included to guide comparisons

moments:

\begin{tabular}{l|c|c|c}
$q \backslash \zeta_{5}$ & $\left\langle x q^{K}\right\rangle$ & $\left\langle x^{2} q^{K}\right\rangle$ & $\left\langle x^{3} q^{K}\right\rangle$ \\
\hline$u$ & $0.19(2)$ & $0.067(09)$ & $0.030(05)$ \\
$\bar{s}$ & $0.23(2)$ & $0.085(11)$ & $0.040(07)$ \\
\hline$u+\bar{s}$ & $0.42(3)$ & $0.152(20)$ & $0.070(12)$
\end{tabular}.

Naturally, the $u$-quark values are unchanged from Eq. (54), but those for the $\bar{s}$-quark are increased by $4.8(8) \%$. The choice $\sigma_{s}=1$ is practically maximal, i.e. if one chooses a value too much larger, then distortions begin to appear at very low $x$ in $\bar{s}^{K}\left(x ; \zeta_{5}\right)$.

The curves in Fig. 13 may be interpolated using the functional form in Eq. (33) and the coefficients and powers in Table 6. These interpolations express the large- $x$ behaviour indicated in Eq. (21), but $q^{K}\left(x ; \zeta_{5}\right) / q_{1}^{K}\left(x ; \zeta_{5}\right)>0.5$ is only realised on $x>0.96$. Here, a result unchanged from Eq. (53):

$\beta_{\text {eff }}\left(\zeta_{5}\right)=2.73(8)$
Our predictions for the kaon's glue and sea DFs are best expressed by the following ratios:

$\mathrm{R}_{\mathscr{g}}^{K \pi}=g^{K}\left(x ; \zeta_{5}\right) / g^{\pi}\left(x ; \zeta_{5}\right)$,

$\mathrm{R}_{\mathcal{S}}^{K \pi}=\mathcal{S}^{K}\left(x ; \zeta_{5}\right) / \mathcal{S}^{\pi}\left(x ; \zeta_{5}\right)$,

which are depicted in Fig. 14. The results drawn therein are described by the curves:

$\begin{aligned} \mathrm{R}_{\mathcal{g}}^{K \pi} & =\frac{1.00-0.877 x}{1-0.828 x} \\ \mathrm{R}_{\mathcal{S}}^{K \pi} & =\frac{1.00-0.473 x}{1-0.228 x}\end{aligned}$

The uncertainty in these ratios owing to that in $\hat{\alpha}(0)$ is negligible, i.e. no larger than the line width in either case.

Evidently, the kaon's glue and sea distributions differ from those of the pion only on the valence region $x \geq 0.2$. In hindsight, this is not surprising: mass-dependent splitting functions act primarily to modify the valence DF of the heavier quark; valence DFs are negligible at low- $x$, where glue and sea distributions are large, and vice versa; hence the biggest impact of a change in the valence DFs must lie at large- $x$. It is notable that each of the predicted ratios depicted in Fig. 14 is pointwise similar to the measured value of $u^{K}\left(x ; \zeta_{5}\right) / u^{\pi}\left(x ; \zeta_{5}\right)$. On the flip side, the glue and sea DFs in the kaon and pion are practically identical on $x \lesssim 0.2$.

Using our computed DFs, we find $\left(\zeta=\zeta_{5}\right)$ :

$\langle x\rangle_{g}^{K}=0.44(2), \quad\langle x\rangle_{\text {sea }}^{K}=0.14(2)$,

with $\langle x\rangle_{\text {sea }_{f}}^{K}=0.091(11),\langle x\rangle_{\text {sea }_{s}}^{K}=0.045(06)$, where $l$ denotes the light-quarks. Comparing these results with those in Eq. (42), then accounting for mass-dependent splitting functions and being careful to use the computed ratio functions, we find that the gluon light-front momentum fraction in the kaon is $\sim 1 \%$ less than that in the pion and the sea fraction is $\sim 2 \%$ less.

It is worth remarking that the impact of the $P_{S \leftarrow g}$ correction in Eq. (59b) is negligible. Eliminating this term changes no result by more than $0.01 \%$. Thus, our analysis indicates that the primary effect of mass-dependent splitting functions is to suppress the transfer of momentum from the heavier valence-quark into glue; and with less glue, there is less sea. One may ignore the additional mass-dependent suppression on the subsequent splitting of gluons into heavier sea quarks introduced by $P_{S \leftarrow g}$.

A comparison with Ref. [103] is now possible. Following Ref. [101], that analysis used algebraic Ansätze for the quark propagators and Bethe-Salpeter amplitudes needed to compute $\pi$ and $K$ valence-quark DFs. The pion analysis was informed by the GRS suggestion [51] that $\left\langle 2 x u^{\pi}\left(x ; \zeta_{0}^{\mathrm{LO}}\right)\right\rangle=$ 0.56 , a value $\gtrsim 20 \%$ smaller than that we find herein (Eq. 31). The valence-quark momentum fraction in the kaon was 
then used as a parameter and fixed in a least-squares fit to the $u^{K} / u^{\pi}$ data in Ref. [119]. The best fit to data was obtained with $\left\langle x\left[u^{K}\left(x ; \zeta_{0}^{\mathrm{LO}}\right)+\bar{s}^{K}\left(x ; \zeta_{0}^{\mathrm{LO}}\right)=0.95(5)\right]\right\rangle$, leading to the conclusion that the valence-quark momentum fraction in the kaon is much larger than that in the pion, or, equivalently, the kaon's glue + sea content is much smaller.

Reviewing the Ref. [103] analysis, our prediction for $u^{K} / u^{\pi}$ in Fig. 12 is best matched by the upper bound on kaon glue + sea determined in Ref. [103], i.e. 10\%. Moreover, any repeat of the Ref. [103] study would now be informed by our results; hence, begin with a significantly smaller glue + sea content in the pion. Thus, one can expect that an update of the phenomenological analysis in Refs. [101,103] would produce results compatible with those described herein.

\section{Summary and perspective}

Working with information gained from solving the continuum meson bound-state problem using sophisticated scattering kernels that incorporate effects generated by the mechanism responsible for the emergence of hadronic mass, we described parameter-free predictions for the leading-twist two-particle distribution amplitudes (DAs) of the $\pi$ - and $K$-mesons (Sects.5.2, 6). Using properties of light-front wave functions, these DAs were then used to provide $\pi$ and $K$ distribution functions (DFs) at the hadronic scale, $\zeta_{H}$ (Sects. 5.2, 7.1). The hadronic scale is fixed by properties of QCD's infrared-finite process-independent effective charge (Sect. 3).

Building upon this foundation and employing an all-orders evolution hypothesis (Sect.4.1), we subsequently delivered predictions for all $\pi$ and $K$ DFs, viz. valence-quark, glue and sea. Focusing first on the pion, we provided comparisons with phenomenological analyses and results from latticeQCD (1QCD) (Sect. 5.3) and arrived at a range of significant conclusions. (i) Amongst existing phenomenological studies of pion structure functions, only one [50] employs a next-toleading-order analysis that includes threshold resummation and this study is unique in producing a valence-quark DF that is consistent with QCD and matches our prediction (Fig. 5). (ii) Our prediction for the pion's valence-quark DF agrees with modern 1 QCD results for the pointwise behaviour of this function. (iii) The general disagreement between phenomenological results and theory predictions for the pion's valence-quark DF feeds into the pion's glue and sea distributions; hence, existing inferences of the pion's gluon distribution disagree with our prediction on $x \lesssim 0.05$ and the pion's phenomenologically determined sea-quark distribution disagrees with our result on the entire physical $x$-domain. A resolution of these conflicts must await improved phenomenological analyses that include threshold resummation and new data that constrains the pion's glue and sea distributions.
Very little empirical information is available on $K$ DFs; so there are no recent phenomenological inferences. Naturally, within QCD, in the absence of Higgs-generated currentquark mass differences, kaon DFs are identical to those of the pion, being entirely determined by the mechanism(s) responsible for emergent hadronic mass (EHM). Hence, all differences between $K$ and $\pi$ DFs result from Higgs-induced modulations of EHM. Regarding kaon valence-quark distributions, there are model calculations and a single, recent 1QCD study; but there are no results for the pointwise behaviour of the kaon's glue and sea distributions. Hence, our predictions for the entire array of kaon DFs currently stand alone. The one piece of available experimental information is the valencequark ratio $u^{K}\left(x ; \zeta_{5}\right) / u^{\pi}\left(x ; \zeta_{5}\right), \zeta_{5}=5.2 \mathrm{GeV}$ [119]. Our prediction for this ratio is consistent with the data (Figs. 10, 12). However, given the large empirical errors, this ratio is very forgiving of even material differences between various calculations of the individual DFs used to produce the ratio. Modern, precise data is critical if this ratio is to be used as a path to understanding the Standard Model's NambuGoldstone modes; and results for $u^{\pi}\left(x ; \zeta_{5}\right), u^{K}\left(x ; \zeta_{5}\right)$ separately would be better.

Regarding the kaon's glue and sea distributions, we predicted that they are similar to those in the pion (Sects. 7.2, 7.3). The degree of similarity depends on the interplay between EHM and Higgs-generated current-quark mass differences. Consequently, a detailed comparison requires the use of mass-dependent splitting functions. We therefore introduced a one-parameter model for the relevant splitting functions in order to illustrate the potential impact on kaon DFs of the mass difference between $u$ and $s$-quarks. This led to the following conclusions: (i) the light-front momentum fraction carried by $s$-quarks in the kaon increases by $\sim 5 \%$; and (ii) this is compensated by a commensurate decrease in the fractions carried by glue $(-1 \%)$ and sea $(-2 \%)$. Our predictions for the $K / \pi$ ratios of glue and sea distributions are presented in Fig. 14.

The analysis described herein can be improved in two ways: (a) one could further test the assumption of factorisation made for the meson light-front wave functions (Sect. 2); and (b) a more rigorous treatment of mass-dependence in splitting functions should be implemented (Sect.7.3). Both improvements are underway.

The Standard Model's (pseudo-) Nambu-Goldstone modes - pions and kaons - are basic to the formation of everything, from nucleons to nuclei, and on to neutron stars. Hence, new-era experiments capable of discriminating between the results and predictions discussed herein should have high priority. Naturally, the phenomenological methods needed to proceed from data to DFs must match modern experiments in precision. Concerning theory, continuum and lattice analyses of the pion's valence-quark DF are converging on the same form, confirming the longstanding QCD expectation, 
Eq. (1); but given the predictions presented herein, lattice results for the pion's glue and sea distributions would be very valuable. This is even more true for the kaon. With only one extant lattice study of kaon DFs, addressing solely the valence distributions, disagreeing in many ways with continuum predictions and also conflicting with Eq. (1), many opportunities are available.

Acknowledgements We are grateful for constructive comments from V. Andrieux, P. Barry, W.-C. Chang, C. Chen, O. Denisov, J. Friedrich, W. Melnitchouk, C. Mezrag, W.-D. Novak, S. Platchkov, M. Quaresma and C. Quintans; and for the hospitality and support of RWTH Aachen University, III. Physikalisches Institut B, Aachen - Germany. Work supported by: National Natural Science Foundation of China (under Grant No. 11805097), Jiangsu Provincial Natural Science Foundation of China (under Grant No. BK20180323). Jiangsu Province Hundred Talents Plan for Professionals; Alexander von Humboldt Foundation; Spanish Ministry of Science and Innovation (MICINN), under Grant No. PID2019-107844GB-C22; and Junta de Andalucía, contract no. P18-FRJ-1132.

Data Availability Statement This manuscript has no associated data or the data will not be deposited. [Authors' comment: All data generated during this study are represented in this published article.]

Open Access This article is licensed under a Creative Commons Attribution 4.0 International License, which permits use, sharing, adaptation, distribution and reproduction in any medium or format, as long as you give appropriate credit to the original author(s) and the source, provide a link to the Creative Commons licence, and indicate if changes were made. The images or other third party material in this article are included in the article's Creative Commons licence, unless indicated otherwise in a credit line to the material. If material is not included in the article's Creative Commons licence and your intended use is not permitted by statutory regulation or exceeds the permitted use, you will need to obtain permission directly from the copyright holder. To view a copy of this licence, visit http://creativecomm ons.org/licenses/by/4.0/.

Funded by SCOAP ${ }^{3}$.

\section{Appendix A: Large $x$ behaviour of meson distribution functions}

In order to establish Eq. (21), consider that in some sufficiently compact neighbourhood $x \simeq 1$ :

$q(x ; \zeta)=(1-x)^{\beta(\zeta)} \sum_{i=0}^{\infty} c_{i}(\zeta) x^{i}$

namely, $q(x ; \zeta) /(1-x)^{\beta(\zeta)}$ has a regular Taylor expansion on this neighbourhood and

$C(\zeta):=\lim _{x \rightarrow 1} \frac{q(x ; \zeta)}{(1-x)^{\beta(\zeta)}}=\sum_{i=0}^{\infty} c_{i}(\zeta)<\infty$.

This being the case, then

$$
\left\langle x^{s} q(x ; \zeta)\right\rangle=\int_{0}^{1} d x x^{s} q(x ; \zeta)
$$

$$
\begin{aligned}
& \stackrel{x \cong 1}{=} \sum_{i=0}^{\infty} c_{i}(\zeta) \int_{0}^{1} d x x^{i+s}(1-x)^{\beta(\zeta)} \\
& =\sum_{i=0}^{\infty} c_{i}(\zeta) \Gamma(1+\beta(\zeta)) \frac{\Gamma(1+i+s)}{\Gamma(2+i+s+\beta(\zeta))} \\
& \stackrel{s \gg 1}{=} \frac{1}{s} \frac{1}{s^{\beta(\zeta)}} \mathcal{C}(\zeta) \Gamma(1+\beta(\zeta))[1+\mathrm{O}(1 / s)] .
\end{aligned}
$$

Consequently,

$$
\begin{aligned}
& \ln \frac{\left\langle x^{s} q(x ; \zeta)\right\rangle}{\left\langle x^{s} q\left(x ; \zeta_{0}\right)\right\rangle} \stackrel{s \gg 1}{=}-\left[\beta(\zeta)-\beta\left(\zeta_{0}\right)\right] \ln s \\
&+\ln \frac{C(\zeta)[1+\beta(\zeta)]}{C\left(\zeta_{0}\right)\left[1+\beta\left(\zeta_{0}\right)\right]}+\mathrm{O}(1 / s)
\end{aligned}
$$

At this point, recall Eqs.(17)-(19), which yield

$$
\begin{aligned}
& \ln \frac{\left\langle x^{s} q(x ; \zeta)\right\rangle}{\left\langle x^{s} q\left(x ; \zeta_{0}\right)\right\rangle}=\frac{\gamma_{0}^{s}}{4 \pi} \int_{\ln \zeta^{2}}^{\ln \zeta_{H}^{2}} d t \hat{\alpha}(t) \\
& s \gg 1
\end{aligned}
$$

where $\gamma_{E}$ is the Euler constant.

Finally, equating like leading- $s$ coefficients in Eqs. (A.4), (A.5b), one arrives at the anticipated results:

$$
\begin{aligned}
& \beta(\zeta)=\beta\left(\zeta_{0}\right)+\frac{3}{2} \chi^{1}\left(\zeta_{0}, \zeta\right), \\
& \mathcal{C}(\zeta)=\mathcal{C}\left(\zeta_{0}\right) \frac{\Gamma\left(1+\beta\left(\zeta_{0}\right)\right)}{\Gamma(1+\beta(\zeta))}\left[\chi^{1}\left(\zeta_{0}, \zeta\right)\right]^{\frac{3}{2}\left[\frac{3}{4}-\gamma_{E}\right]}
\end{aligned}
$$

A similar derivation can be found in Ref. [18, Sect. VIII].

\section{References}

1. G. Aad et al., Phys. Lett. B 716, 1 (2012)

2. S. Chatrchyan et al., Phys. Lett. B 716, 30 (2012)

3. J. Ellis, Phil. Trans. Roy. Soc. Lond. A 370, 818 (2012)

4. H.A. Chawdhry, M.A. Lim, A. Mitov, Phys. Rev. D 99, 076011 (2019)

5. P. Hägler, Phys. Rept. 490, 49 (2010)

6. C. Alexandrou, Prog. Part. Nucl. Phys. 67, 101 (2012)

7. H.-W. Lin et al., Prog. Part. Nucl. Phys. 100, 107 (2018)

8. L. Chang, C.D. Roberts, P.C. Tandy, Chin. J. Phys. 49, 955 (2011)

9. C.D. Roberts, J. Phys. Conf. Ser. 706, 022003 (2016)

10. G. Eichmann, H. Sanchis-Alepuz, R. Williams, R. Alkofer, C.S. Fischer, Prog. Part. Nucl. Phys. 91, 1 (2016)

11. C.S. Fischer, Prog. Part. Nucl. Phys. 105, 1 (2019)

12. M.S. Bhagwat, L. Chang, Y.-X. Liu, C.D. Roberts, P.C. Tandy, Phys. Rev. C 76, 045203 (2007)

13. A.C. Aguilar et al., Eur. Phys. J. A 55, 190 (2019)

14. C.D. Roberts, Few Body Syst. 58, 5 (2017) 
15. W. Bentz, T. Hama, T. Matsuki, K. Yazaki, Nucl. Phys. A 651 , 143 (1999)

16. A.E. Dorokhov, L. Tomio, Phys. Rev. D 62, 014016 (2000)

17. R. Davidson, E. Ruiz Arriola, Acta Phys. Polon. B 33, 1791 (2002)

18. E. Ruiz Arriola, W. Broniowski, Phys. Rev. D 70, 034012 (2004)

19. J. Lan, C. Mondal, S. Jia, X. Zhao, J.P. Vary, Phys. Rev. D 101, 034024 (2020)

20. J.M. Cornwall, Phys. Rev. D 26, 1453 (1982)

21. D. Dudal et al., JHEP 01, 044 (2004)

22. P.O. Bowman et al., Phys. Rev. D 70, 034509 (2004)

23. E. Luna, A. Martini, M. Menon, A. Mihara, A. Natale, Phys. Rev. D 72, 034019 (2005)

24. A. Aguilar, D. Binosi, J. Papavassiliou, Phys. Rev. D 78, 025010 (2008)

25. J. Rodríguez-Quintero, JHEP 1101, 105 (2011)

26. P. Boucaud et al., Few Body Syst. 53, 387 (2012)

27. S. Strauss, C.S. Fischer, C. Kellermann, Phys. Rev. Lett. 109, 252001 (2012)

28. D. Binosi, L. Chang, J. Papavassiliou, C.D. Roberts, Phys. Lett. B 742, 183 (2015)

29. A.C. Aguilar, D. Binosi, J. Papavassiliou, Front. Phys. China 11, 111203 (2016)

30. F. Siringo, Nucl. Phys. B 907, 572 (2016)

31. A.K. Cyrol, L. Fister, M. Mitter, J.M. Pawlowski, N. Strodthoff, Phys. Rev. D 94, 054005 (2016)

32. F. Gao, S.-X. Qin, C.D. Roberts, J. Rodríguez-Quintero, Phys. Rev. D 97, 034010 (2018)

33. D. Binosi, R.-A. Tripolt, Phys. Lett. B 801, 135171 (2020)

34. A. Aguilar, D. Binosi, J. Papavassiliou, J. Rodríguez-Quintero, Phys. Rev. D 80, 085018 (2009)

35. D. Binosi, C. Mezrag, J. Papavassiliou, C.D. Roberts, J. Rodríguez-Quintero, Phys. Rev. D 96, 054026 (2017)

36. J. Rodríguez-Quintero, D. Binosi, C. Mezrag, J. Papavassiliou, C.D. Roberts, Few Body Syst. 59, 121 (2018)

37. J. Rodríguez-Quintero, L. Chang, K. Raya, C. D. Roberts, Process-independent effective coupling and the pion structure function. In: 27th International Nuclear Physics Conference (INPC 2019) Glasgow, Scotland, July 29-August 2, 2019 (2019). arXiv:1909.13802 [nucl-th]

38. Z.-F. Cui et al., Chin. Phys. C 44, 083102 (2020)

39. M. Ding et al., Chin. Phys. (Lett.) 44, 031002 (2020)

40. M. Ding et al., Phys. Rev. D 101, 054014 (2020)

41. Y.L. Dokshitzer, Sov. Phys. JETP 46, 641 (1977)

42. V.N. Gribov, L.N. Lipatov, Sov. J. Nucl. Phys. 15, 438 (1972)

43. L.N. Lipatov, Sov. J. Nucl. Phys. 20, 94 (1975)

44. G. Altarelli, G. Parisi, Nucl. Phys. B 126, 298 (1977)

45. Z.F. Ezawa, Nuovo Cim. A 23, 271 (1974)

46. G.R. Farrar, D.R. Jackson, Phys. Rev. Lett. 35, 1416 (1975)

47. E.L. Berger, S.J. Brodsky, Phys. Rev. Lett. 42, 940 (1979)

48. R.J. Holt, C.D. Roberts, Rev. Mod. Phys. 82, 2991 (2010)

49. K. Wijesooriya, P.E. Reimer, R.J. Holt, Phys. Rev. C 72, 065203 (2005)

50. M. Aicher, A. Schäfer, W. Vogelsang, Phys. Rev. Lett. 105, 252003 (2010)

51. M. Glück, E. Reya, I. Schienbein, Eur. Phys. J. C 10, 313 (1999)

52. P.C. Barry, N. Sato, W. Melnitchouk, C.-R. Ji, Phys. Rev. Lett. 121, 152001 (2018)

53. M. Oehm et al., Phys. Rev. D 99, 014508 (2019)

54. B. Joó et al., Phys. Rev. D 100, 114512 (2019)

55. R.S. Sufian et al., Phys. Rev. D 99, 074507 (2019)

56. S.J. Brodsky, G.P. Lepage, Adv. Ser. Direct. High Energy Phys. 5, $93(1989)$

57. S.J. Brodsky, H.-C. Pauli, S.S. Pinsky, Phys. Rept. 301, 299 (1998)

58. T. Heinzl, Lect. Notes Phys. 572, 55 (2001)

59. G. 't Hooft, Nucl. Phys. B 75, 461 (1974)

60. L. Chang et al., Phys. Rev. Lett. 110, 132001 (2013)
61. H.J. Munczek, Phys. Rev. D 52, 4736 (1995)

62. A. Bender, C.D. Roberts, L. von Smekal, Phys. Lett. B 380, 7 (1996)

63. L. Chang, I.C. Cloët, C.D. Roberts, S.M. Schmidt, P.C. Tandy, Phys. Rev. Lett. 111, 141802 (2013)

64. K. Raya et al., Phys. Rev. D 93, 074017 (2016)

65. T. Horn, C.D. Roberts, J. Phys. G. 43, 073001 (2016)

66. M. Chen, M. Ding, L. Chang, C.D. Roberts, Phys. Rev. D 98 091505(R) (2018)

67. P. Maris, C.D. Roberts, Phys. Rev. C 56, 3369 (1997)

68. P. Maris, P.C. Tandy, Phys. Rev. C 60, 055214 (1999)

69. G. Eichmann, R. Alkofer, A. Krassnigg, D. Nicmorus, Phys. Rev. Lett. 104, 201601 (2010)

70. G. Eichmann, Phys. Rev. D 84, 014014 (2011)

71. H. Sanchis-Alepuz, G. Eichmann, S. Villalba-Chavez, R. Alkofer, Phys. Rev. D 84, 096003 (2011)

72. Q.-W. Wang, S.-X. Qin, C.D. Roberts, S.M. Schmidt, Phys. Rev. D 98, 054019 (2018)

73. S.-X. Qin, C.D. Roberts, S.M. Schmidt, Few Body Syst. 60, 26 (2019)

74. C. Shi et al., Phys. Lett. B 738, 512 (2014)

75. C. Shi et al., Phys. Rev. D 92, 014035 (2015)

76. L. Chang, C.D. Roberts, Phys. Rev. Lett. 103, 081601 (2009)

77. F. Gao, L. Chang, Y.-X. Liu, C.D. Roberts, P.C. Tandy, Phys. Rev. D 96, 034024 (2017)

78. M. Ding et al., Phys. Rev. D 99, 014014 (2019)

79. G.P. Lepage, S.J. Brodsky, Phys. Lett. B 87, 359 (1979)

80. A.V. Efremov, A.V. Radyushkin, Phys. Lett. B 94, 245 (1980)

81. G.P. Lepage, S.J. Brodsky, Phys. Rev. D 22, 2157 (1980)

82. C. Mezrag et al., Phys. Lett. B 741, 190 (2015)

83. S.-S. Xu, L. Chang, C.D. Roberts, H.-S. Zong, Phys. Rev. D 97, 094014 (2018)

84. R.L. Jaffe, G.G. Ross, Phys. Lett. B 93, 313 (1980)

85. R.K. Ellis, W.J. Stirling, B.R. Webber, $Q C D$ and Collider Physics (Cambridge University Press, Cambridge, UK, 2011)

86. G. Grunberg, Phys. Rev. D 29, 2315 (1984)

87. Y.L. Dokshitzer, Perturbative QCD theory (includes our knowledge of $\alpha$ (s)) - hep-ph/9812252, in High-Energy Physics. Proceedings, 29th International Conference, ICHEP'98, Vancouver, Canada, 23-29 July 1998, Vols. 1, 2, pp. 305-324 (1998)

88. Y.L. Dokshitzer, D.E. Kharzeev, Ann. Rev. Nucl. Part. Sci. 54, 487 (2004)

89. A. Deur, S.J. Brodsky, G.F. de Teramond, Prog. Part. Nucl. Phys. 90, 1 (2016)

90. P. Hoyer, Bound states and QCD. arXiv:1807.05598 [hep-ph]

91. S.-S.Xu et al., Newperspective on hybridmesons. Eur. Phys. J. A (Lett.) 55, 113 (2019)

92. E.V. Souza et al., Eur. Phys. J. A (Lett.) 56, 25 (2020)

93. M. Ding, F. Gao, L. Chang, Y.-X. Liu, C.D. Roberts, Phys. Lett. B 753, 330 (2016)

94. N. Chouika, C. Mezrag, H. Moutarde, J. Rodríguez-Quintero, Phys. Lett. B 780, 287 (2018)

95. D. Binosi et al., Phys. Lett. B 790, 257 (2019)

96. K. Raya, M. Ding, A. Bashir, L. Chang, C.D. Roberts, Phys. Rev. D 95, 074014 (2017)

97. Y.-Z. Xu et al., Phys. Rev. D 100, 114038 (2019)

98. A. Deur, V. Burkert, J.-P. Chen, W. Korsch, Phys. Lett. B 650, 244 (2007)

99. A. Deur, V. Burkert, J.-P. Chen, W. Korsch, Phys. Lett. B 665, 349 (2008)

100. S.J. Brodsky, G.F. de Teramond, A. Deur, Phys. Rev. D 81, 096010 (2010)

101. L. Chang et al., Phys. Lett. B 737, 23 (2014)

102. M.B. Hecht, C.D. Roberts, S.M. Schmidt, Phys. Rev. C 63, 025213 (2001) 
103. C. Chen, L. Chang, C.D. Roberts, S. Wan, H.-S. Zong, Phys. Rev. D 93, 074021 (2016)

104. D. Westmark, J.F. Owens, Phys. Rev. D 95, 056024 (2017)

105. I. Novikov et al., (2020), Parton distribution functions of the charged pion within the xFitter framework. arXiv:2002.02902 [hep-ph]

106. J. Lan, C. Mondal, S. Jia, X. Zhao, J.P. Vary, Light Meson Parton Distribution Functions from Basis Light-Front Quantization and QCD Evolution. In: 18th International Conference on Hadron Spectroscopy and Structure. arXiv:2003.12249 [hep-ph]

107. P.J. Sutton, A.D. Martin, R.G. Roberts, W.J. Stirling, Phys. Rev. D 45, 2349 (1992)

108. O. Denisov et al. Letter of Intent (Draft 2.0): A New QCD facility at the M2 beam line of the CERN SPS. arXiv: 1808.00848 [hep-ex]

109. W.-C. Chang, J.-C. Peng, S. Platchkov, T. Sawada, Constraining Gluon Density of Pions at Large $x$ by Pion-induced $J / \psi$ Production. arXiv:2006.06947 [hep-ph]

110. J.T. Londergan, G.Q. Liu, E.N. Rodionov, A.W. Thomas, Phys. Lett. B 361, 110 (1995)

111. J.S. Conway et al., Phys. Rev. D 39, 92 (1989)

112. G. Altarelli, Phys. Rept. 81, 1 (1982)

113. H. Georgi, H.D. Politzer, Phys. Rev. D 9, 416 (1974)

114. D. Gross, F. Wilczek, Phys. Rev. D 9, 980 (1974)

115. H.D. Politzer, Phys. Rept. 14, 129 (1974)

116. V. Braun et al., Phys. Rev. D 74, 074501 (2006)
117. R. Arthur et al., Phys. Rev. D 83, 074505 (2011)

118. J. Segovia et al., Phys. Lett. B 731, 13 (2014)

119. J. Badier et al., Phys. Lett. B 93, 354 (1980)

120. H.-W. Lin, J.-W. Chen, Z. Fan, J.-H. Zhang, R. Zhang, The Valence-Quark Distribution of the Kaon from Lattice QCD. arXiv:2003.14128 [hep-lat]

121. F. Martin, A.I.P. Conf, Proc. 68, 797 (1981)

122. M. Gluck, E. Reya, M. Stratmann, Eur. Phys. J. C 2, 159 (1998)

123. M. Alberg, J. Tibbals, Phys. Lett. B 709, 370 (2012)

124. D. Adikaram, et al. Measurement of Tagged Deep In-elastic Scattering (TDIS), approved Jeerson Lab experiment E12-15-006 (2015)

125. J. Annand, et al. Measurement of Kaon Structure Func-tion through Tagged Deep Inelastic Scattering (TDIS), approved Jeerson Lab experiment C12-15-006A (2017)

126. L.D. Landau, I. Pomeranchuk, Dokl. Akad. Nauk Ser. Fiz. 92, 535 (1953)

127. A.B. Migdal, Phys. Rev. 103, 1811 (1956)

128. S. Catani, Z. Phys. C 75, 665 (1997)

129. S.M. Kim, Mod. Phys. Lett. A 16, 467 (2001)

130. C. Pascaud, Generalisation of DGLAP equations to massive partons. arXiv:1111.3262 [hep-ph]

131. F. Gao et al., private communication in preparation 